\title{
Queueing Models for Computer Communications System Analysis
}

\author{
HISASHI KOBAYASHI, SENIOR MEMBER, IEEE, AND ALAN G. KONHEIM
}

\author{
Invited Paper
}

\begin{abstract}
Modeling and performance prediction are becoming increasingly important issues in the design and operation of computer communications systems. Complexities in their configuration and sophistications in resource sharing found in today's computer communications demand our intensive effort to enhance the modeling capability. The present paper is intended to review the state of affairs of analytic methods, queueing analysis techniques in particular, which are essential to modeling of computer communication systems. First we review basic properties of exponential queueing systems, and then give an overview of recent progress made in the areas of queueing network models and discrete-time queueing systems. A unified treatment of buffer storage overflow problems will be discussed as an application example, in which we call attention to the analogy between buffer behavior and waiting time in the $G I / G / 1$ queue. Another application deals with the analysis of various multiplexing techniques and network configuration. An extensive reference list of the subject fields is also provided.
\end{abstract}

\section{INTRODUCTION}

$\mathbf{T}$ THE field of computer communications has witnessed rapid growth and technological innovations in recent years. By the term computer communications we loosely imply a variety of user-to-computer, or computer-to-computer interfaces realized by communication links. They range from various forms of teleprocessing seen in today's data-processing industry, time-sharing systems between collections of terminals and central computers, to the burgeoning computer-tocomputer communication networks typified by the Advanced Research Projects Agency Network.(ARPANET). A number of books and articles have been written on various aspects of this - increasingly important subject area. The reader is referred, for example, to books by Abramison and Kuo [2], Chu [20], Davies and: Barber [33], Green and Lucky [52], Grimsdale and Kuo [55], Martin [105], and articles by Green and Tang [33], Schwartz, Boorstyn, and Pickholtz [137] among many others.

With the increasing complexity and sophistication of computer communication systems, modeling and performance evaluation are becoming critical issues in the design and operation of such systems. It is apparent that for a cost-effective design we must be equipped with systematic methods of predicting quantitative relations between system resource parameters, system workloads, and measures of system performance. Any computer communication system is, in essence, composed of an organized collection of resources-hardware, soitware; and combinations thereof-which are usually shared by multiple users, messages, or processes. The sharable resources are for instance, communication links, computational re-

Manuscript received April 5, 1976; revised August 4, 1976.

The authors are with the IBM Thomas J. Watson Research Center, Yorktown Heights, NY 10598. sources of remotely located computers, file systems, and data bases in a distributed system.

The communication links are a major cost component in a data-transmission system. In addition to the conventional multiplexing methods, such as frequency-division multiplexing (FDM) and synchronous time-division multiplexing (STDM), a number of new forms of channel sharing have been proposed to achieve more efficient and flexible usage of communication media by a large number of terminal-based users. The most successful development in this direction is a random-access multiplexing method which has been devised and implemented by the ALOHA system project at the University of Hawaii (see Abramson [1], Kuo and Binder [99]). This multiplexing technique, known as the ALOHA technique or ALOHA channel, is particularly suited to a packet communication system which uses a radio or satellite channel. Another form of statistical and dynamic sharing of a channel is the asynchronous timedivision multiplexing (ATDM) technique studies by Chu [21], [22], Rudin [134], and others.

Multiplexing of a central computer is the key idea of timesharing systems, which have grown tremendously in their number and sophistication within the last decade. Many of today's computing systems which support the teleprocessing applications are quite complicated; they are often multiprogrammed virtual-storage sytems. More frequently than not, a teleprocessing system is one of the many application subsystems built on a common machine and operating system. Scheduling and allocation of resources of a central processing system (not just the sharing of CPU time, but sharing of all of the system resources including memory, auxiliary storage, devices, and supporting hardware) is certainly a major component in any computer communication system. Modeling of a computer 'system/subsystem has been pursued by a great number of people, and recent results on analytic models are found, for example, in Kleinrock. [78], Kobayashi [86], and in a number of references cited therein.

The most recent development in computer communications is the increasing interest and development efforts found in intercomputer-communication nets such as the ARPANET and its descendents. In a computer network the primary resources to be shared are geographically separated computers (often called host computers), the program library, and the data bases attached to them. In order to support efficient and flexible sharing of these resources, such novel techniques as messageswitching and packet-switching multiplexing have been introduced, at the expense of additional complexity of system structure: that is, a set of message-switched processors [called interface message processors (IMP's)] now constitutes the highest level of the network below which groups of hosts exist and are attached to the corresponding IMP's. As a con- 
sequence, buffer spaces of the individual IMP's, as well as message-switching processors themselves and communication links connected to them, contribute to the list of critical resources to be considered in the design and operation of the network.

Whenever there is a sharing of a scarce resource, contention for the resource will arise. Flows of messages or data generated by user terminals, multiplexers, concentrators, or by host computers, are not steady streams. On the contrary, occurrences of messages are often sporadic and bursty. The amount of resource usage demanded by the messages or processes is often unpredictable and consequently is viewed as stochastic in nature; for example, the length of messages or data to be transferred or stored, the time required in processing transactions, the amount of main memory or buffer pool to be allocated to processes. In intercomputer-communication nets another dimension is added to the stochastic nature of the work loads; namely, the destination IMP's or hosts of messages are also assumed to be random variables, and we face new issues such as routing and flow control of messages.

Because of the unpredictable nature of the work demands placed on the resources, congestion occurs (occasionally or frequently, depending upon the work load level), queues will be formed, and delays introduced at critical resources. In the performance analysis of a computer communication system/ subsystem, we must therefore take these queues into consideration. One of the major issues which concerns designers of complex information-processing systems is the lack of capability to predict performance measures such as response time, throughput, and resource utilization. A problem of similar nature will be faced by those who configurate (see Green and Tang [53]) a system, given a set of candidate components or subsystems, such as communication links of various capacities, central processors of different speeds, memory and buffer storage of different capacities, various types of topologies connecting terminals to local processors. Of course, performance prediction is not the only major technical issue in design of computer-communication systems. However, such well discussed issues as routing and flow control, link capacity assignment, concentrator placement, allocation and distribution of data base, are certainly not separable from the performance analysis. The problem of link capacity assignment, for example, has been successfully formulated by Kleinrock [74] and others as an optimization or mathematical programming problem based on the average delay formula derived from queueing analysis. How to schedule and allocate resources effectively among competing requests is certainly in the realm of congestion or queueing theory in a broader sense. Therefore, it is clear that queueing models provide a basic framework and the mathematical tools for dealing with a large class of system design issues. Several other disciplines of applied mathematics, such as graph theory, mathematical programming, optimization techniques, and reliability theory must augment the queueing analysis in order to cope with the overall design and analysis issues. A survey article by Green and Tang [53] addresses a number of the specific technical problems that concern professionals engaged in design, configuration, and maintenance of teleprocessing systems.
The intent of the present paper is to review that portion of queueing theory which is relevant to modeling and performance evaluation of computer communication systems and subsystems. Of course there are a number of circumstances in which the currently available techniques cannot provide a satisfactory solution. In such circumstances, a simulation model is often the only viable alternative. Even when a decision is made for simulation, an analytic solution, however crude it may be, should be sought for. An analytic model can serve as a guideline in narrowing down a range of system configurations and parameters under which a simulation runs. It also could save a considerable amount of modeling efforts, by detecting possible errors introduced in the design and implementation phases of a simulator. There are a large number of books and articles on queueing theory: Bhat [7], Cox and Smith [31], Feller [39], Gnedenko and Kovalenko [48], Kleinrock [79], Newell [113], Riordan [129], and Syski [143]. Due to space limitations, our treatment is not as broad as some readers might wish to see. An extensive bibliography at the end (and the books cited above) will hopefully direct the reader to fundamental and collateral reading. For the same reason, our presentation does not discuss many specific applications, instead focusing on fundamental and general methodologies.

In Section II, we briefly review the important properties of the Poisson process and its generalizations-the nonstationary Poisson process, the compound Poisson process, and the renewal process. The Poisson process plays an important role in congestion theory, in much the same way as does the Gaussian process in conventional communication and information theory. The Gaussian process and the Poisson process are related in the sense that both occur as the limiting distribution obtained when one superimposes independent processes; the former in amplitude, the latter in epochs on the time axis. It is an empirically and analytically established fact that a Poisson process model provides a quite acceptable mathematical abstraction of message traffic in many computer communication systems.

Section III discusses a class of queueing systems which we have designated as exponential systems. This class includes the so-called birth-and-death process model for a single-server system, and queueing networks of exponential servers.

Section IV is devoted to a discussion of generalized Markovian queueing network models. A "network of queues" model is capable of representing asynchronous parallel operations and mutual interactions of separate resources, such as observed in central processing units and channels [or input/ output (I/O) processors] in a multiprogrammed computer system and in a set of message switching processors within a computer network. Kleinrock [80] successfully applied an exponential queueing network model to the analysis of the ARPANET. There has been noteworthy progress in recent years in enlarging the class of queueing network models for which we may find closed form (analytic) solutions for the queue length distribution in equilibrium. The key results will be highlighted in Section IV.

The literature of queueing theory deals largely with continuous-time models-the arrival of requests and their demands 
are allowed to assume real values. In communications systems it appears more natural to formulate the problems within a discrete-time framework. In Section V we discuss how various results of the continuous-time model can be transferred into the discrete-time setting. We consider, as an example, a model of buffer storage which should find direct applications in such problems as the design of buffered terminals and the study of storage allocation strategies in multiplexing and concentration. We will derive asymptotically tight upper and lower bounds for the buffer overflow probability and other related quantities, based on the recent results obtained for $G I / G / 1$ queues. The relations between these bounds and a result recently reported by Wyner [151] will also be discussed.

Section VI proceeds to the analysis of various topological structures and their associated schemes for multiplexing and concentration. Our formulation is general enough to encompass such typical connections as the star, loop, and multidrop networks. A unified treatment is developed for the analysis of various disciplines of time-division multiplexing: STDM, ATDM, polling, and chaining.

Section VII is a concluding section in which we give brief discussions of a number of important topics and results. We shall identify some of the research subjects which require continues and extensive investigations in the future.

As discussed above, queues for service of one kind or another arise in many different parts of computer communication systems; a queue of terminal users waiting for processing by another host; a queue of messages waiting for buffer storage allocation at a message switching node (e.g., IMP) in a store-and-forward network, etc. The unit of operational flow through a queueing system is usually called a customer in the traditional language of queueing theory. In a computer communication system a customer is typically a message, packet, job, transaction, etc. In some cases we may model terminal users, or even host systems, as customers depending on the congestion problem with which we are dealing. A server or service station may be a transmission line, channel, host system, IMP, multiplexer, etc.

A customer is drawn from a population or input source. One characterization of the population is its size. It may be assumed to be either infinite or finite. Since a mathematical model tends to be far more tractable for the infinite population, this assumption is often made even when the actual size is some relatively large finite number. Another characteristic of the demands upon a system is the arrival pattern (or process) by which customers or messages are generated over time. Many authors assume that the arrival of customers is described by a Poisson process which we will discuss in Section II.

A finite population model is generally more complicated analytically, because the number of customers already in the queueing system at any point in time affects the number of potential customers remaining in the input source. In some cases, however, we are forced to assume a finite population if the rate at which the input source generates arrivals is significantly affected by the number of customers in the queueing system. Care must be exercised in the selection of a population model. Use of infinite population models sometimes leads to a false conclusion; for example, that the modeled system is un- stable (unable to process customers at a rate commensurate with their entry into the system) although the actual system to be modeled is not. Although it will be discussed later, it seems worthwhile to note here that a queueing system with a finite population source can be conveniently modeled as a closed queueing network.

\section{THE POISSON PROCESS}

The Poisson process plays a central role in the statistical modeling of many physical phenomena. We shall give a brief account of its principal properties. Details can be found in the excellent monograph of Khintchine [70].

By a counting process $\mathbf{N}=\left\{N_{t}: 0 \leqslant t<\infty\right\}$ we mean a family of nonnegative-integer-valued random variables $N_{t}$ which are nondecreasing in (time) $t . N_{t}$ counts the accumulated number of events in the time interval $(0, t]$. It is usual to set $N_{0}=0$, assuming that initially there are no counted events. If $0<t_{1}<t_{2}$, the increment of $N_{t}$ over the interval $\left(t_{1}, t_{2}\right]$, denoted by $N_{\left(t_{1}, t_{2}\right]}$, is this change in $N_{t}, N_{t_{2}}-N_{t_{1}}$, over the interval. Within our context, an event might be the arrival of a message in a computer-communications system. The average number of events in $(0, t]$ (the expected value of $\left.N_{t}\right)$ is called the principal function of the process $\mathbf{N}$ and is denoted by $\Lambda(t)=E\left\{N_{t}\right\} .1$

A counting process may satisfy one or more of the following three properties.

Stationary Increments: If the intervals $\left\{\left(a_{i}, b_{i}\right]: 1 \leqslant i \leqslant n\right\}$ are nonoverlapping,

$$
0 \leqslant a_{1}<b_{1} \leqslant a_{2}<b_{2} \leqslant \cdots \leqslant a_{n}<b_{n}<\infty,
$$

then for every $h>0$, the two $n$-dimensional vector-valued random variables (of increments)

$$
\left(N_{\left(a_{1}, b_{1}\right]}, \cdots, N_{\left(a_{n}, b_{n}\right]}\right)
$$

and

$$
\left(N_{\left(a_{1}+h, b_{1}+h\right]}, \cdots, N_{\left(a_{n}+h, b_{n}+h\right]}\right)
$$

have the same distribution.

Independent Increments: If the intervals $\left\{\left(a_{i}, b_{i}\right]: 1 \leqslant i \leqslant n\right\}$ are nonoverlapping, then random variables $\left\{N_{\left(a_{i}, b_{i}\right]}: 1 \leqslant i \leqslant\right.$ $n\}$ are independent.

Orderliness: $\operatorname{Pr}\left\{N_{t+h}-N_{t}>1\right\}=o(h)$ as $h \rightarrow 0 .{ }^{2}$ If $N$ has stationary increments, it can be shown that the limit,

$$
\lim _{h \rightarrow 0}(1 / h) \operatorname{Pr}\left\{N_{t+h}-N_{t}=1\right\}=\lambda
$$

exists with $0 \leqslant \lambda \leqslant \infty ; \lambda$ is the rate of the counting process.

\section{Remarks:}

1) Stationarity of the increments means that the process $\mathbf{N}$ loses track of absolute time. For $h>0$, the shifted process

${ }^{1}$ The expected value of a random variable $X$ will be denoted by $E\{X\}$ and the variance by $\operatorname{var}\{X\}$.

${ }^{2}$ A function $f(h)$ is of order $o(h)$ as $h \rightarrow 0$ if $f(h) / h \rightarrow 0$ as $h \rightarrow 0$. 
$\mathbf{N}^{h}=\left\{N_{t+h}-N_{h}: 0 \leqslant t<\infty\right\}$, which counts events after time $h$, is a replica of the original process.

2) If $\mathbf{N}$ is orderly, then the probability of more than one event in $(t, t+h], \operatorname{Pr}\left\{N_{t+h}-N_{t}>1\right\}$, is "small" compared to $h$ for "small" $h$. In general this probability is not equal to 0 .

An orderly counting process with stationary and independent increments is called a Poisson process. Its principal function is $\Lambda(t)=\lambda t$ where $\lambda$ is the rate of the process. One can prove for this process that the state probabilities $\left\{p_{k}(t)\right\}$ are given by

$$
p_{k}(t)=\operatorname{Pr}\left\{N_{t}=k\right\}=(\lambda t)^{k} e^{-\lambda t} / k ! \quad 0 \leqslant k<\infty .
$$

The generating function of this Poisson process (or more precisely the generating function of the state probabilities $\left\{p_{k}(t)\right\}$ of $\left.(1)\right)$ is the function of the complex variable $z$ defined by

$$
E\left\{z^{N} t\right\}=\sum_{0 \leqslant k<\infty} p_{k}(t) z^{k}=\exp (\lambda t(z-1)) .
$$

Let $\left\{T_{i}: 1 \leqslant i<\infty\right\}$ be the epochs at which events occur in the Poisson process and set $\tau_{i}=T_{i}-T_{i-1}\left(1 \leqslant i<\infty ; T_{0}=0\right\}$. The sets

$$
\left\{N_{t} \geqslant n\right\}=\left\{T_{n} \leqslant t\right\}=\left\{\tau_{1}+\cdots+\tau_{n} \leqslant t\right\}
$$

provide three equivalent descriptions of the statement-at least $n$ events have occurred in the interval $(0, t]$. For the Poisson process one may show that the random variables $\left\{\tau_{i}\right\}$ are independent and identically distributed (iid) with the common exponential distribution

$$
\operatorname{Pr}\left\{\tau_{i} \leqslant t\right\}= \begin{cases}0, & -\infty<t<0 \\ 1-\exp (-\lambda t), & 0 \leqslant t<\infty .\end{cases}
$$

The $\left\{\tau_{i}\right\}$ are the interarrival times of events and $E\left\{\tau_{i}\right\}=1 / \lambda$.

There are two operations-joining and splitting-which we may perform on Poisson processes. If $\left\{\mathbf{N}^{(i)}\right\}$ are independent Poisson processes with rates $\left\{\lambda^{(i)}\right\}$, then the join of these processes is the process $\mathbf{N}=\Sigma_{i} \mathbf{N}^{(i)}$ which counts the events which occur in any of the processes. The join $\mathbf{N}$ has rate $\lambda=$ $\Sigma_{i} \lambda^{(i)}$. Conversely, starting with a Poisson process $\mathbf{N}$ of rate $\lambda$ and a $\vartheta$ in $[0,1]$ we may split $\mathbf{N}$ into $N^{(0)}$ and $N^{(1)}$. As each event of $\mathbf{N}$ occurs we toss a coin; if a head results (with probability $\vartheta$ ) we mark this event as an event of $N^{(1)}$ while if a tail occurs the event is marked as an event of $N^{(0)}$. The two counting processes $\mathbf{N}^{(i)}(i=0,1)$ thus obtained are independent and Poisson of rates $(1-\vartheta) \lambda$ and $\vartheta \lambda$, respectively.

The Poisson process has been generalized in many ways. Three are worth taking note of because of their possible applicability in describing the flow of messages in a communications system.

\section{Nonstationary Poisson Processes}

If $\mathbf{N}$ is orderly, has independent increments, and if the limit

$$
\lim _{h \rightarrow 0}(1 / h) \operatorname{Pr}\left\{N_{t+h}-N_{t}=1\right\}=\lambda(t)
$$

exists, then $\mathbf{N}$ is a nonstationary Poisson process and

$$
\begin{aligned}
p_{k}\left(t_{1}, t_{2}\right)= & \operatorname{Pr}\left\{N_{\left(t_{1}, t_{2}\right]}=k\right\} \\
=\left(\Lambda\left(t_{2}\right)-\Lambda\left(t_{1}\right)\right)^{k} \exp \left(-\left(\Lambda\left(t_{2}\right)-\Lambda\left(t_{1}\right)\right) / k !\right. & 0<k<\infty
\end{aligned}
$$

where $\Lambda(t)=\int_{0}^{t} \lambda(u) d u$. A simple computation shows that $\Lambda(t)$ is the principal function of the process $N$. The function $\Lambda(t)$ is nonnegative, nondecreasing, and right-continuous with $\Lambda(0)=0$. Its derivative $\lambda(t)$ may be interpreted as an instantaneous rate of the nonstationary process.

Conversely, starting with a nondecreasing, right-continuous, nonnegative function $\Lambda(t)$ we may construct a nonstationary Poisson process whose state probabilities are given by (4). The function $\Lambda(t)$ need not be differential nor even continuous. The points of discontinuity of $\Lambda(t)$, those points $t_{0}$ at which $\Lambda\left(t_{0}\right)-\Lambda\left(t_{0}-\right)>0$, correspond to time at which events are certain to take place. For example, if in some physical process an "event" is certain to take place at 5:12 P.M., then we might choose to incorporate this phenomena in our construction of the principal function. A nonstationary processes might be used to model phenomena which have a pronounced variability with time whose effects we wish to understand.

\section{Compound Poisson Processes}

The property of orderliness insures that events are "simple," i.e., the probability of a multiple event in a small interval is negligible with respect to the length of the interval. If we model the arrival of messages in a computer communications systems, the counting process may represent the number of data units arriving where a data unit may be byte, character, or block of characters of fixed length (a packet). In such cases we may wish to allow for the arrival of multiple data units (packets) at a time. Let $\mathrm{A}=\left\{a_{k}: 1 \leqslant k<\infty\right\}$ be a probability distribution for the length of a message if $\mathbf{N}$ is a Poisson process with rate $\lambda$ we may construct a compound Poisson process $\mathbf{N}[\mathbf{A}]$ by the superposition of the processes. $\mathbf{N}$ will determine the times of the events and $A$ the number of events which take place. The generating function of the state probabilities of the compound process $\mathbf{N}[\mathbf{A}]$ is

$$
E\left\{z^{N[A]} t\right\}=\exp \{\lambda t(\Phi(z)-1)\}
$$

where

$$
\Phi(z)=\sum_{1 \leqslant k<\infty} a_{k} z^{k} .
$$

At this point it may be appropriate to consider the question, "Why Poisson processes?" A major reason for assuming that a phenomenom can be described by a Poisson process is the analytical simplicity that often follows from this hypothesis. A justification of the Poisson hypothesis may be possible since the Poisson law is the limit law of certain families of random variables. The most elementary limit theorem of this type is the Poisson approximation to Bernoulli trials. Suppose $\left\{X_{n, i}: 1 \leqslant i \leqslant n\right\}$ represents the $n$th trial of an experiment; on the $n$th trial we toss a coin $n$ times with outcomes $X_{n, i}=1$ if the $i$ th toss was a head and 0 if a tail. Let the probability of 
head be $p_{n}$ so that the expected number of successes (heads) on the $n$th trial is $n p_{n}$. If $p_{n}$ varies with $n($ as $n \rightarrow \infty)$ so that $n p_{n} \rightarrow \lambda$, then

$$
\sum_{1 \leqslant i \leqslant n} X_{n, i} \rightarrow \text { Poisson law }(\lambda), \quad n \rightarrow \infty
$$

The convergence in (6) is convergence in law; that is, the distribution function of the random variables $\Sigma_{i} X_{n, i}$, $\operatorname{Pr}\left\{\Sigma_{i} X_{n, i} \leqslant k\right\}$ converges to the Poisson distribution function $\sum_{j \leqslant k} \lambda^{j} \exp (-\lambda) / j !$. More generally suppose that $\left\{X_{n, i}\right\}$ are random variables (assuming nonnegative integer values) satisfying

$$
\begin{aligned}
& \operatorname{Pr}\left\{X_{n, i}=k\right\}=a_{n, i, k} \\
& \sum_{1 \leqslant i \leqslant n} \sum_{k>1} a_{n, i, k} \rightarrow 0, \quad n \rightarrow \infty \\
& \sum_{1 \leqslant i \leqslant n} a_{n, i, 1} \rightarrow \lambda, \quad n \rightarrow \infty .
\end{aligned}
$$

Then $\Sigma_{1 \leqslant i \leqslant n} X_{n, i} \rightarrow$ Poisson law. Further details can be found in Feller [39], Gnedenko and Kovalenko [48], and Loeve [102].

An interpretation of the above mathematical argument in the context of our applications is possible: in many cases the message or transaction generation of individual terminal users or host systems can be described by stationary renewal processes or more general point processes. These component processes can generally be regarded as statistically independent. Then by virtue of the above limit theorem we can show that the total traffic is "close" to a Poisson process if the number of independent processes is "large" while the intensity of each of the components is "small." Empirical studies by Fuchs and Jackson [43] and Lewis and Yue [100] of certain computer communication traffic suggest that the Poisson model provides reasonable agreement with actual measurement data.

Our final generalization of the Poisson process starts from the previous observation that the interarrival times $\left\{\tau_{i}\right\}$ of events are exponentially distributed. A renewal process is a sequence of nonnegative iid random variables $\mathbf{T}=\left\{\tau_{i}: 1 \leqslant\right.$ $i<\infty\}$. In the language of renewal theory, $\tau_{i}$ is a "lifetime," the lifetime of a lightbulb, the time to failure of a machine, the interarrival time between messages in a communication system. Starting with a renewal process we may construct a counting process $\mathrm{N}=\left\{N_{t}\right\} ; N_{t}$ will be the number of lifetimes observed up to time $t$, that is, the number of lightbulbs replaced, the number of machine failures, the number of messages which have arrived. $N_{t}$ is defined by $N_{t}=\max \left\{n: \tau_{1}+\right.$ $\left.\tau_{2}+\cdots+\tau_{n} \leqslant t\right\}$. The Poisson process is the counting process constructed from the renewal process of exponentially distributed lifetimes. For the Poisson process we have $E\left\{N_{t}\right\}=$ $t / E\left\{\tau_{i}\right\}=\lambda t$. This equality does not hold for the general renewal process. The main theorem of renewal theory replaces this equality by the limit theorem, $\lim _{t} N_{t} / t=1 / E\left\{\tau_{i}\right\}$. For further details see the book by Cox [29] and the fundamental paper of Smith [138].
Another component of a queueing system is the characterization of the amount of service required by an individual customer or message, which we call the service demand, or simply, service or work. The unit of service or work varies depending upon the nature of the server and customer. If the server is a transmission line or channel, an appropriate unit will be [bit], [byte], [character], [packet]. If the server is a host computer system and customers or jobs require service by a processor then the appropriate unit may be [ínstruction]. In the modeling of a system we assume that the demands are random variables. In most cases we shall consider, the population will be homogeneous and the service demands of customers $\left\{W_{i}\right\}$ will be iid random variables.

The arrival process and service demands are descriptions of the customers. We must now specify the service rate or processing rate of the server. In the present paper we denote the service rate by $C$ since this quantity corresponds to the line capacity if the server is a transmission line, as in most of our applications. Thus the units of $C$ are [bits $/ \mathrm{s}],[\mathrm{kbits} / \mathrm{s}]$. This quantity is sometimes called the bandwidth of the channel or line, since $C$ is proportional to the effective bandwidth.

If the service demand variable $W$ [work units] and the processing rate $C$ [work units/second] are given, their ratio

$$
S=\frac{W}{C} \text { [seconds] }
$$

is a random variable called the service time. The sequence $\left\{S_{i}\right\}$ of the service times of customers is called the service process. The inverse of the expectation of the service time

$$
\mu=\frac{1}{E\{S\}}=\frac{C}{E\{W\}}\left[\text { seconds }^{-1}\right]
$$

is called the (job) completion rate in the queueing literature. If $C$ is a constant, there is no essential need for distinguishing between service demand $W$ and service time $S$. Thus we often set $C=1$ by default and represent the service demand in terms of the service time.

In some cases, however, the processing rate is not a constant, but varies depending upon the "state" of the service station. Such an example is a multiserver queue in which $s$ servers constitute a service station sharing a common waiting line. If the processing rates of the individual servers are all $C$, and the number of customers currently in the station is $i$, then the total service.rate of the station is given by

$$
C(i)=\min (i, s) C
$$

which in turn gives the queue-dependent job completion rate

$$
\mu_{i}=\min (i, s) \mu
$$

Another example of queue dependent processing rate $C(i)$ is found when the effective processing rate degrades as the system congestion increases. 


\section{EXPONENTIAL SYSTEMS}

We use the term exponential system in referring to queueing systems in which the arrival and service processes have exponential distributions. Important results are due to Burke Reich, and Jackson. The simplest exponential system is the single server queue (Fig. 1) which is referred to as the $M / M / 1$ system. For the $M / M / 1$ system the arrival process is Poisson and the service process is exponential. By this we mean that the interarrival times between events (or customers) $\left\{\tau_{i}: 1 \leqslant\right.$ $i<\infty\}$ and the service times $\left\{S_{i}: 1 \leqslant i<\infty\right\}$ satisfy the following conditions. 1) The $\left\{\tau_{i}\right\}$ are iid random variables with $F(t)=$ $\operatorname{Pr}\left\{\tau_{i} \leqslant t\right\}=1-e^{-\lambda t}(0 \leqslant t<\infty)$. 2) The service times $\left\{S_{i}\right\}$ are iid with $G(t)=\operatorname{Pr}\left\{S_{i} \leqslant t\right\}=1-e^{-\mu t}(0 \leqslant t<\infty)$. 3) The service and arrival processes are independent. To complete the specification of the $M / M / 1$ queueing system we must describe the manner in which the customers are served by the service facility; this is called the queue discipline. The simplest such discipline is order of arrival ("first-come-first-served," FIFO). All of the results in the present section are valid for a larger class of service disciplines called work-conserving (Kleinrock [75], Wolff [148]). A queue discipline is said to be workconserving, if 1) the service demand $W$ of each job is not affected by the queue discipline, 2) the queue discipline does not take advantage of knowledge about service demands (or times) and/or arrival times of the individual jobs, and 3) the server is not idle when there are jobs waiting for service. Certainly this class includes such disciplines as FIFO, LIFO, random, round-robin (RR), foreground-background or feedback (FB), and processor sharing (PS). The state of the system at time $t$ is the random process $X=\left\{X_{t}: 0 \leqslant t<\infty\right\}$ where $X_{t}$ is the number of customers either in service or waiting for service. Because of the memoryless property of the exponential distribution, this description of the state of the system defines a Markov process. Let $\pi_{i, j}(t)$ denote the transition probabilities

$$
\pi_{i, j}(t)=\operatorname{Pr}\left\{X_{t}=j / X_{0}=i\right\}, \quad 0 \leqslant i, j<\infty
$$

and $\Pi(t)$ the matrix $\left(\pi_{i, j}(t)\right)$. The transition probabilities satisfy two systems of differential equations, called the forward and backward equations.

$$
\begin{array}{ll}
(d / d t) \Pi(t)=\Pi(t) Q \quad & (\text { forward } \text { equation }) \\
(d / d t) \Pi(t)=Q \Pi(t) & \text { (backward equation) }
\end{array}
$$

where $Q=\left(q_{i, j}\right)$ is the matrix

$$
q_{i, j}= \begin{cases}-\left[\lambda+\mu \chi_{(i>0)}\right], & \text { if } j=i \\ \lambda, & \text { if } j=i+1 \\ \mu \chi_{(i>0)}, & \text { if } j=i-1 \\ 0, & \text { if } j \neq i-1, i, i+1 .\end{cases}
$$

\footnotetext{
${ }^{3} x_{E}$ denotes the characteristic or indicator function of the event or condition $E . x_{E}$ equals 1 if the condition holds and 0 otherwise. This provides a simple notation avoiding the writing of special formulas for certain index conditions.
}

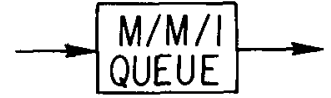

Fig. 1. $M / M / 1$ queue.

$Q$ is called the infinitesimal generator or transition rate matrix of the process $X$. The matrix equations (7) and (8) may be written in perhaps the more familiar form

$$
\begin{aligned}
(d / d t) \pi_{i, j}(t)= & -\left[\lambda+\mu \chi_{(j>0)}\right] \pi_{i, j}(t) \\
& +\lambda \chi_{(j>0)} \pi_{i, j-1}(t)+\mu \pi_{i, j+1}(t) \\
(d / d t) \pi_{i, j}(t)= & -\left[\lambda+\mu \chi_{(i>0)}\right] \pi_{i, j}(t) \\
& +\lambda \pi_{i+1, j}(t)+\mu \chi_{(i>0)} \pi_{i-1, j}(t) .
\end{aligned}
$$

The derivation of the forward (10) and backward (11) equations employs a standard and important technique. For the forward equation the change in state $i \rightarrow j$ which takes place over the interval $(0, t+h]$ is classified according to the possible transitions in the interval $(t, t+h]$ for "small" $h$. There are three possibilities, as follows.

1) The state at time $t$ is $j$ and no change takes place in $(t$, $t+h]$.

2) The state at time $t$ is $j-1$ and an arrival takes place in $(t, t+h]$.

3) The state at time $t$ is $j+1$ and a service completion takes place in $(t, t+h]$.

The exponential nature of the arrival and service processes imply that the probability of more than one "change" ( $m$ arrivals and $n$ service completions with $m+n>1)$ in $(t, t+h]$ is $o(h)$ as $h \rightarrow 0$. Thus

$$
\begin{aligned}
\pi_{i, j}(t+h)= & {\left[1-\left(\lambda+\mu \chi_{(j>0)}\right) h+o(h)\right] \pi_{i, j}(t) } \\
& +\left(\lambda \dot{\chi}_{(j>0)}(t) h+o(h)\right) \pi_{i, j-1}(t) \\
& +(\mu h+o(h)) \pi_{i, j+1}(t) .
\end{aligned}
$$

Rearranging (12), dividing by $h$ and letting $h \rightarrow 0^{+}$we obtain (10). The derivation of the backward equation (11) employs the same technique, only now the state transitions in the interval $(0, h]$ are examined.

The transition laws $\Pi(t)=\left(\pi_{i, j}(t)\right)$ together with an initial distribution $\left\{\xi_{i}=\operatorname{Pr}\left\{X_{0}=i\right\}\right\}$ of the state at time 0 determine the process $X$. The state probability $p_{j}(t)=\operatorname{Pr}\left\{X_{t}=\right.$ $j$ \} is given by

$$
p_{j}(t)=\sum_{i} \xi_{i} \pi_{i, j}(t)
$$

If the process $\mathbf{X}$ is irreducible, i.e., for all $i$ and $j, \pi_{i, j}(t)>0$ for some $t$, then $\lim _{t} p_{j}(t)=p_{j}$ exists. These limits define the stationary distribution of the process. It may be that $p_{j}=0$ for all $j$ (the transient case). In our applications, the interesting case will be when the $\left\{p_{j}\right\}$ defines a bona-fide probability distribution. These probabilities then satisfy the equations 


$$
\begin{aligned}
& p Q=0 \\
& Q p=0
\end{aligned}
$$

where $\boldsymbol{p}=\left(p_{0}, p_{1}, \cdots\right)$. Foster's theorem [40] states that if a vector $\boldsymbol{p}=\left(p_{0}, p_{1}, \cdots\right)$ exists satisfying

$$
\begin{aligned}
& p Q=0 \\
& \sum_{i}\left|p_{i}\right|<\infty,
\end{aligned}
$$

then a stationary distribution exists and the vector $\boldsymbol{p}$ will have strictly positive components. In this case $X$ is an ergodic Markov chain and the state probabilities $\left\{p_{j}(t)\right\}$

$$
p_{j}(t)=\sum_{i} p_{i} \pi_{i, j}(t)
$$

are independent of $t$; that is, $p_{j}(t)=p_{j}$. To verify the independence with time note that ( 8 ) implies

$$
\sum_{i} p_{i}(d / d t) \pi_{i, j}(t)=\sum_{i, k} p_{i} q_{i, k} \pi_{k, j}
$$

The right-hand side of (18) is zero by (14) and hence $(d / d t) p_{j}(t)=0$, as was to be proved. This of course justifies the term stationary!

The stationary distribution $\left\{p_{i}\right\}$ for the $M / M / 1$ system is easily found. The state probabilities $p_{j}(t)=\operatorname{Pr}\left\{X_{t}=j\right\}$ satisfy the differential equations

$$
\begin{aligned}
(d / d t) p_{j}(t)= & -\left[\lambda+\mu \chi_{(j>0)}\right] p_{j}(t) \\
& +\lambda \chi_{(j>0)} p_{j-1}(t)+\mu p_{j+1}(t) .
\end{aligned}
$$

Equation (19) follows directly from (10) when we multiply it by $\xi_{i}$ and sum over $i$. If the limit, $p_{j}=\lim _{t \rightarrow \infty} p_{j}(t)$ is to exist, it follows that the derivative of $p_{j}(t)$ converges to 0 . Thus (19) reduces to the steady-state equations

$$
\left[\lambda+\mu \chi_{(j>0)}\right] p_{j}=\lambda \chi_{(j>0)} p_{j-1}+\mu p_{j+1} .
$$

We now verify that

$$
p_{j}=\rho^{j}(1-\rho)
$$

is a solution to (20) with $\rho=\lambda / \mu$ provided $\rho<1$. The ratio $\rho$ is called the utilization of the system. It is easy to prove that

$$
\rho=\lim _{T \rightarrow \infty}(1 / T) \int_{0}^{T} \chi_{\left(X_{u}>0\right)} d u
$$

so that $\rho$ may be interpreted as the limiting fraction of time the state is nonzero, that is, the fraction of time the server is busy (or being utilized).

In the modeling of computer-communications systems we encounter service systems composed of many service facilities which may be linked together in special geometries. The output of an $M / M / 1$ queue may form the input to other stations in the service network. What is the nature of the output process? Reich [125] and Burke [8] were the first to study the nature of the output process of an $M / M / 1$ queue. The Reich-Burke theorem is stated as follows.

Theorem: The output process of an $M / M / 1$ queueing system, in which 1) the initial distribution is the stationary distribution, and 2) the service is according to the discipline order of arrival, is Poisson. Moreover, the state of the system $X_{t}$ and the number of departures in $(0, t]$ are independent.

Note that the conclusion remains valid for work-conserving disciplines. The theorem implies that with an arbitrary initial distribution, the output process converges in law as $t \rightarrow \infty$ to the Poisson distribution.

The output theorem, as Burke refers to the above result, holds in somewhat more generality. A Markov process $\mathbf{X}=$ $\left\{X_{t}: 0 \leqslant t<\infty\right\}$ in which the state space (the set of values taken by the $\left.X_{t}\right)$ is the nonnegative integers $\{0,1,2, \cdots$,$\} is$ called a birth and death process provided that the only infinitesimal changes allowed are

$$
i \rightarrow i+1 \quad \text { (birth) }
$$

and

$$
i \rightarrow i-1 \quad \text { (death). }
$$

By infinitesimal changes we mean precisely that the conditional probabilities satisfy

$$
\begin{aligned}
\operatorname{Pr} & \left\{X_{t+h}=j / X_{t}=i\right\} \\
& = \begin{cases}1-\left[\mu_{i}+\lambda_{i}\right] h+o(h), & \text { if } j=i \\
\mu_{i} h+o(h), & \text { if } j=i-1 \\
\lambda_{i} h+o(h), & \text { if } j=i+1 \\
o(h), & \text { if } j \neq i-1, i, i+1 .\end{cases}
\end{aligned}
$$

The parameters $\left\{\mu_{i}, \lambda_{i}\right\}$ are called the death and birth rates. Some examples follow.

M/M/1 Queue

$$
\begin{aligned}
& \lambda_{i}=\lambda \\
& \mu_{i}= \begin{cases}\mu, & \text { if } i>0 \\
0, & \text { if } i=0 .\end{cases}
\end{aligned}
$$

$M / M / s$ Queue-s Servers

$$
\begin{aligned}
& \lambda_{i}=\lambda \\
& \mu_{i}=\min (i, s) \mu .
\end{aligned}
$$

$M / M$ with Processor Sharing

$$
\begin{aligned}
& \lambda_{i}=\lambda \\
& \mu_{i}= \begin{cases}\mu / i, & \text { if } i>0 \\
0, & \text { if } i=0 .\end{cases}
\end{aligned}
$$

$$
\begin{aligned}
M / M / \infty & \\
\lambda_{i} & =\lambda \\
\mu_{i} & =i \mu, \quad i<\infty .
\end{aligned}
$$

For a birth and death process set 


$$
\begin{aligned}
\pi_{0} & =1 \\
\pi_{j} & =\frac{\lambda_{0} \lambda_{1} \cdots \lambda_{j-1}}{\mu_{1} \mu_{2} \cdots \mu_{j}} .
\end{aligned}
$$

The birth and death process $\left\{X_{t}\right\}$ possesses a stationary distribution, that is,

$$
p_{k}=\lim _{t \rightarrow \infty} \operatorname{Pr}\left\{X_{t}=k\right\}>0
$$

exists and is strictly positive provided

$$
\sum_{j} \pi_{j}<\infty
$$

and when this condition is met

$$
p_{j}=\pi_{j} / \sum_{i} \pi_{i}
$$

Equation (30) and the stability condition (29) provide the formulas for the four queueing models mentioned as well as for other models of $M / M$-type systems where the service rate is state-dependent.

The output theorem is generalized as follows: if $\mathbf{X}$ is a birth and death process satisfying: 1) $\lambda_{i}=\lambda$;2) $\Sigma_{j} \pi_{j}<\infty$; and 3) the initial distribution $\operatorname{Pr}\left\{X_{0}=j\right\}$ is given by (30), then the sequence of death times forms a Poisson process of rate $\lambda$ and the number of deaths in $(0, t]$ and the state at time $t$ are independent.

As the proof is so elementary we include it for the reader's convenience. Let $a_{k, j}(t)$ be the probability of $k$ deaths in $(0, t]$ and the state at time $t+0$ be $j$. Then by considering possible infinitesimal changes in $(t, t+h]$ we have

$$
\begin{aligned}
a_{k, j}(t+h)= & {\left[1-\left(\lambda+\mu_{j}\right) h+o(h)\right] a_{k, j}(t) } \\
& +\mu_{j+1} h a_{k-1, j+1}(t)+\lambda h a_{k, j-1}(t)+o(h)
\end{aligned}
$$

which yields the differential equation

$$
\begin{aligned}
(d / d t) a_{k, j}(t)= & -\left[\lambda+\mu_{j}\right] a_{k, j}(t) \\
& +\mu_{j+1} a_{k-1, j+1}(t)+\lambda a_{k, j-1}(t) .
\end{aligned}
$$

It is now easy to show (by direct substitution) that $a_{k, j}(t)=$ $p_{j} b_{k}(t)$ is a solution of (32) where $p_{j}$ is the stationary probability distribution of the process [see (30)] and $b_{k}(t)=$ $(\lambda t)^{k} e^{-\lambda t} / k !$.

The output theorem has a very interesting consequence in the study of networks of $M / M$-type queues-the so-called Markovian networks. Consider the simplest such Markovian network consisting of two service stations connected in series or in tandem (Fig. 2).

Customers enter this network at the first node (or stage), queue for service and upon completion of service immediately join the queue at the second node. The state of the system is the two-dimensional vector-valued random process $X=\left\{X_{t}=\right.$

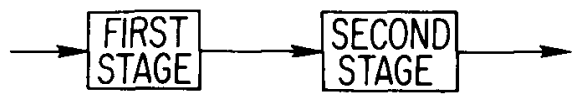

Fig. 2. Two-stage tandem queueing network.

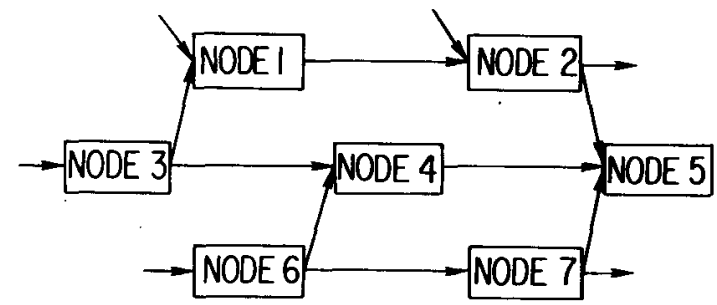

Fig. 3. Service network.

$\left.\left(X_{t, 1}, X_{t, 2}\right): 0 \leqslant t<\infty\right\}$ where $X_{t, i}$ is the number of customers queued or in service at the $i$ th stage. If 1 ) the arrival process is Poisson (with rate $\lambda$ ), 2) the service processes are exponential with rates $\left.\mu_{i}(i=1,2), 3\right)$ the queue discipline in each stage is work-conserving, and 4) the service and arrival processes are independent, then the output process of the first node will be asymptotically Poisson (as $t \rightarrow \infty$ ) and we may apply the analysis for the $M / M / 1$ queue to obtain the joint distribution of the number of customers waiting (or in service) at each stage obtaining

$$
p_{i, j}=\lim _{t \rightarrow \infty} \operatorname{Pr}\left\{X_{t}{ }^{\prime}=(i, j)\right\}=\rho_{1}{ }^{i} \rho_{2}{ }^{j}\left(1-\rho_{1}\right)\left(1-\rho_{2}\right)
$$

where

$$
\rho_{i}=\lambda / \mu_{i}, \quad i=1,2 .
$$

See Muntz [109] for further discussion on this subject.

The solution for the state probabilities (33) of the tandem network was generalized by Jackson [65]. Start with a graph $G$ of nodes and edges; nodes represent service stations and edges paths linking the stations (Fig. 3).

Customers enter the system at the nodes; the external arrival process at node $n_{i}$ is a Poisson process of rate $\lambda_{i}$. The $i$ th node is a service station with $s_{i}$ servers each offering service according to the exponential distribution with parameter $\mu_{i}$. After service is completed at node $n_{i}$, the customer either immediately joins the queue at the $j$ th node with probability $\theta_{i, j}$ or leaves the system with probability $\theta_{i}=1-\Sigma_{j} \theta_{i, j}$. The flow into $\mathbf{n}_{i}$ is composed of: 1 ) the external flow with rate $\lambda_{i}$; and 2$)$ the flow from node $j(1 \leqslant j \leqslant n)$. If we denote by $\Gamma_{i}$ the net flow into $\mathbf{n}_{i}$, then this second flow must have rate $\Gamma_{j} \theta_{j, i}$ since the proportion $\theta_{j, i}$ of the output of $\mathbf{n}_{j}$ is diverted to the input of $\mathbf{n}_{i}$. The flows $\left\{\Gamma_{i}\right\}$ must then satisfy

$$
\Gamma_{j}=\lambda_{j}+\sum_{i} \Gamma_{i} \theta_{i, j}
$$

The state of the network is described by the $n$-dimensional vector-valued random process

$$
\mathrm{X}=\left\{X_{t}=\left(X_{t, 1}, X_{t, 2}, \cdots, X_{t, n}\right): 0 \leqslant t<\infty\right\}
$$

where $n$ is the number of nodes and $X_{t, i}$ is the number of 
customers queued or in service at node $\boldsymbol{n}_{i}$ at time $t$. Let $p_{k}(t)=\operatorname{Pr}\left\{X_{t}=k\right\}$ be the state probability. Jackson [65] proved that if $\Gamma_{i}<s_{i} \mu_{i}(1 \leqslant i \leqslant n)$, then

$$
p_{k}=\lim _{t \rightarrow \infty} p_{k}(t)=\prod_{1 \leqslant i \leqslant n} p_{i, k_{i}}
$$

where $p_{i, k j}$ is the stationary solution for the probability of the queue size being $k_{i}$ in the $M / M / s_{i}$ system with arrival process Poisson of rate $\Gamma_{i}$. Equation (36) suggests that these individual components of the network of Fig. 3 act independently and as if their input processes were Poisson with rates $\left\{\Gamma_{i}\right\}$.

First, let us consider equation (35). When does it have a solution? Let $\Theta$ be the matrix $\left(\theta_{i, j}\right)$. Assume that for some $n$, $\Theta^{n}$ has row sums bounded away from 1 . This implies that after some number of state transitions $i \rightarrow j$ there is a positive probability that a customer will depart from the system. This condition suffices to guarantee the existence of a positive solution to (35). The matter of independence is a much deeper question. If as before $a_{k, j}(t)$ is the probability that: 1) there have been $\boldsymbol{k}$ departures in $(0, t]\left(k_{i}\right.$ from $\left.\left.\mathbf{n}_{i}\right) ; 2\right)$ the state at time $t$ is $j$, then $a_{k, j}(t)$ is not the product of the state probability (36) and a Poisson distribution. The output theorem does not hold in this case even though the state probability (36) has the form which would result if it were true.

Jackson's result assumes that the rate of service at node $\mathbf{n}_{\boldsymbol{i}}$ depends only upon the state of the queue at that node. In many systems the service rate at a node is influenced by the state of other nodes. For example, in the tandem network of Fig. 2 we may admit the possibility of blocking. The server in the first stage will be blocked whenever $M$ or more requests are queued or in service at the second stage. Obtaining the stationary state probabilities for Markovian networks operating under various blocking disciplines poses formidable analytical problems. For the two-stage tandem network (Fig. 2) these probabilities are not separable as in the Jackson model. Konheim and Reiser [96] give a closed-form solution for the exponential case (exponentially distributed service times and Poisson arrivals); earlier results may be found in a paper of Neuts [110].

Gordon and Newell [51] considered a variant of the Jackson model in which the total number of customers (messages) is fixed. The state of this closed network may be described as in Jackson's model by the $n$-dimensional vector-valued random process $\mathbf{X}=\left\{\boldsymbol{X}_{t}=\left(X_{t, 1}, \cdots, X_{t, n}\right)\right\}$. In the GordonNewell model the state space (the set of values assumed by the variables $\left.X_{t}\right)$ is the set $\left\{\boldsymbol{k}=\left(k_{1}, \cdots, k_{n}\right): k_{i} \geqslant 0, \Sigma_{i} k_{i}=M\right\}$ where $M$ is the number of customers (messages) allowed in the system. The closed model is particularly appealing when modeling an $\mathrm{I} / \mathrm{O}$-processor configuration in which case $M$ is the degree of multiprogramming. Since the random process $\mathbf{X}$ has a finite state space, the general theory of Markov chains assumes that it has a stationary distribution provided that the process is irreducible. Gordon and Newell proved that, like Jackson's result, the stationary distribution is of "product" form

$$
p_{k}=\lim _{t \rightarrow \infty} p_{k}(t)=\lim _{t \rightarrow \infty} \operatorname{Pr}\left\{X_{t}=k\right\}=C \prod_{1 \leqslant i \leqslant n} p_{i, k_{i}}
$$

where $p_{i, k_{j}}=\rho_{i}{ }^{k_{i}}\left(1-\rho_{i}{ }^{k_{i}}\right)$ and the normalizing constant $C$ is chosen so that the probabilities in (37), when summed over the state space, equal unity. In general the determination of the normalizing constant $C$ is not straightforward. The parameters $\left\{\rho_{i}\right\}$ may be quite general functions of $i$ and the number of states large. The numerical aspects of the determination of the normalization constant has been examined by Reiser and Kobayashi [127], [128] and Buzen [10].

One final remark; in computer communications applications in which messages are transmitted between nodes in a network, the service time depends upon 1) the length of the message and 2) the line speed. Evidently the service time of the same message at different nodes is dependent. We have pretended that these service times are chosen independently for the same message! Indeed not only are the service times dependent but the interarrival time between this message (when it enters externally into a node) and the next message are dependent. Kleinrock [74] recognized this defect in the modeling and called the assumption we make in the analysis the message independence condition. It is claimed that there is evidence to support the viability of this assumption [80] .

\section{GENERALIZATION OF MARKOVIAN NETWORKS}

The class of Markovian networks defined in Section III have been substantially enlarged in recent years. We will highlight these results in this section. For detailed derivations see Chandy [12], Baskett et al. [6], Kobayashi and Reiser [88], Muntz [109], Kobayashi [86], and Kleinrock [79].

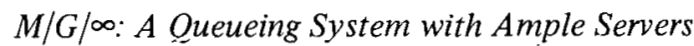

Start with time epochs $\left\{\tau_{n}\right\}$ distributed as a Poisson process at rate $\lambda$, and a set of iid random variables $\left\{S_{n}\right\}$ which are independent of the $\left\{\tau_{n}\right\}$ and have a common general distribution (with distribution function $G$ ). Doob [34] was the first to note that the set of points $\left\{\tau_{n}+S_{n}\right\}$ also constitute a Poisson process of rate $\lambda$. In the queueing theoretic context, this property means that the output process of the queueing system $M / G / \infty$ is a Poisson process with the same rate as that of the input. ${ }^{4}$ Mirasol [108] showed that for a Poisson arrival process, the number of jobs in an $M / G / \infty$ system has a Poisson distribution with mean at time $t$

$$
\lambda \int_{0}^{t}(1-G(x)) d x
$$

where $G$ is the service time distribution. Hence in the equilibrium state, the mean is

$$
\rho=\lambda E\{S\}=\lambda \int_{0}^{\infty}(1-G(x)) d x
$$

The above result can be further extended to those cases in which both arrival and service rate are queue-dependent, as discussed in the section on exponential servers. Let $\lambda_{k}$ be the

\footnotetext{
${ }^{4}$ It has also been shown that for a nonhomogeneous Poisson input with rate $\lambda(t)$, the output process is a nonhomogeneous Poisson process with intensity $\int_{0}{ }^{\infty} \lambda(t-u) d G(u)$.
} 
queue-dependent arrival rate and $C_{k}$ the total processing capacity when there are $k$ customers in the $M / G / \infty$ system at any given time. Then we can prove that for arbitrary distribution of work $G_{W}$, the steady-state distribution of the number $k$ of customers waiting or in service is given by the following formula similar to (27)-(30) of the birth-and-death process:

$$
p_{k}=\pi_{k} / \sum_{i} \pi_{i}
$$

where $\pi_{i}$ is defined by (27) in which $\mu_{i}$ is the queue-dependent rate

$$
\mu_{i}=\frac{C_{i}}{E\{W\}}
$$

Here $E\{W\}$ is the mean work demanded by a customer

$$
E\{W\}=\int_{0}^{\infty} x d G_{w}(x)=\int_{0}^{\infty}\left(1-G_{w}(x)\right) d x
$$

The result in (40) allows another representation which we find useful,

$$
p_{k}=p_{0} \beta_{k}(E\{W\})^{k}
$$

where

$$
\beta_{k}=\frac{\lambda_{0} \lambda_{1} \cdots \lambda_{k-1}}{C_{1} C_{2} \cdots C_{k}}
$$

and

$$
p_{\mathbf{0}}^{-1}=\sum_{0 \leqslant k<\infty} \beta_{k}(E\{W\})^{\mathbf{k}}=B(E\{W\}) .
$$

$B$ is the generating function of the sequence $\left\{\beta_{k}\right\}$. If the arrival rate is independent of the state of the system, and the service capacity of the individual server is constant, i.e.,

$$
\lambda_{k}=\lambda, \quad 0 \leqslant k<\infty
$$

and

$$
C_{k}=k C, \quad 1 \leqslant k<\infty
$$

then we have

$$
\beta_{k}=(\lambda / C)^{k} / k !
$$

and

$$
B(z)=\exp (\lambda z / C)
$$

which easily leads to the aforementioned result that the steady-state distribution (43) is a Poisson distribution

$$
p_{k}=\rho^{k} e^{-\rho} / k !
$$

where the mean value $\rho$ is

$$
\rho=\lambda E\{W\} / C=\lambda / \mu \text {. }
$$

When the input rate is a queue-dependent Poisson process with rate $\lambda_{k}$, the output process possesses the following property [109]

$$
\lim _{h \rightarrow 0}(1 / h) \operatorname{Pr}\left\{\text { a departure in }(t-h, t) / X_{t}=k\right\}=\lambda_{k}
$$

where $X_{t}$ is, as defined earlier, the number of customers in the system at time $t$. This property together with the orderliness of the departure process-the rate of multiple departures is zero-shows that the departure process with time reversed is a Poisson process with rate $\lambda_{k}$. If in particular, the arrival process is a state-independent Poisson process, so is the departure process as we have noted earlier. We should note that the property (43) holds for the output process of the general birth-and-death process discussed earlier.

\section{$M / G / 1$ under Processor-Sharing}

The notion of processor-sharing (PS) was initially introduced by Kleinrock [76] as the limiting case of round-robin (RR) scheduling for a time-shared system in which the time quantum (time slice) is allowed to approach zero. Under this discipline, when there are $k$ customers at the service facility each customer receives service at the rate $C_{k} / k$ [work units/s], where $C_{k}$ is the processing capacity of the server. When a customer enters the system, he immediately starts receiving a share of the server. Thus there is no waiting time. This situation is exactly what we observed for the $M / G / \infty$. Thus the solutions for $M / G / 1$-PS resemble those for $M / G / \infty$ : namely the formulas (40)-(45) hold here also. The departure process possesses the corresponding property as that of the $M / G / \infty$ and $M / M / 1$ (birth-and-death process model).

If the processing capacity of the service facility is independent of congestion,

$$
C_{k}=C, \quad \text { for } 1 \leqslant k<\infty
$$

and the arrival rate is independent of $k$, then $\beta_{k}$ of (44) is now simplified to

$$
\beta_{k}=(\lambda / C)^{k}
$$

so that

$$
B(z)=1 /[1-\lambda z / C]
$$

which yields the following steady-state distribution

$$
p_{k}=(1-\rho) \rho^{k} \text {. }
$$

The parameter $\rho$ is the utilization as given by (51). Equation (56) is exactly the same as (21) of the $M / M / 1$ system. In fact we can show under more general conditions that the PS discipline effectively transforms a general service distribution $G_{W}$ with mean $E\{W\}$ into the exponential distribution with the same mean, insofar as the process $X_{t}$ (and hence also with respect to the queue distribution $\left\{p_{k}\right\}$ and departure process) is concerned! For other interesting properties of the PS discipline see Kleinrock [78] and O'Donovan [116]. 


\section{Different Classes of Customers}

Thus far we have assumed a homogeneous customer population. We now introduce different classes of customers. A class$r$ customer $(r=1,2, \cdots, R)$ has a general service distribution $G_{i, r}$ at node (or service station) $i$ within the network of queues. We are assuming that the service station is either 1 ) an ample parallel server (and hence can be modelled as $M / G / \infty$ for Poisson arrivals); or 2) a station under PS.

When the service discipline at node $i$ is FIFO or any other work-conserving queue discipline ${ }^{\mathbf{5}}$ it is necessary to assume a single-class exponential distribution in order that the network enjoy the Markovian property.

The notion of multiclass is also applicable to customer routing behavior in the network. The transition probabilities $\left\{\theta_{i, j}\right\}$ defined in Section III may now be generalized to the set $\left\{\theta_{i, r, j, s}\right\}$ where $\theta_{i, r, j, s}$ is the probability that a class-r customer leaving node $i$ will immediately join node $j$ as a class-s customer.

The state of the network is now to be described by matrixvalued random process

$$
\mathrm{X}=\left\{X_{t}=\left(X_{i, r}\right)_{t}: 1 \leqslant i \leqslant N, 1 \leqslant r \leqslant R, 0 \leqslant t<\infty\right\}
$$

where the matrix $\left(X_{i, r}\right)_{t}$ gives a "snapshot" of the state of the system at time $t$; that is, the number of class-r customers at node $i$ at time $t$. It is important to recognize that $\mathrm{X}$ is not a Markov process if any of the service time distributions is nonexponential. By applying the method of stages (Cox [30], Cox and Smith [31], Kleinrock [79]) the "general" service time distribution can be handled although this requires an augmented state space.

We assume that customers enter externally according to Poisson processes with rates $\lambda_{K}$ where $K$ is the matrix

$$
K=\left(k_{i, r}\right) .
$$

The joint distribution of $X_{t}=\left(X_{i, r}\right)_{t}$ is

$$
\begin{aligned}
p_{\boldsymbol{K}} & =\operatorname{Pr}\left\{X_{t}=K\right\} \\
& =p_{\mathbf{0}} \Lambda(|| K||) \prod_{1 \leqslant i \leqslant N} \gamma_{i, k_{i}} k_{i} ! \prod_{1 \leqslant r \leqslant R}\left(e_{i, r} E\left\{W_{i, r}\right\}\right)^{k_{i, r}}
\end{aligned}
$$

where

$$
\begin{aligned}
& k_{i}=\sum_{r} k_{i, r} \quad|| K||=\sum_{i} k_{i} \\
& \Lambda(k)=\prod_{0 \leqslant i<k} \lambda_{i}
\end{aligned}
$$

and

$$
\gamma_{i, k_{i}}=1 /\left(C_{i, 1} \times C_{i, 2} \times \cdots \times C_{i, k_{i}}\right) .
$$

$\lambda_{n}$ is the total external arrival rate to the network when the network population is $n=\|K\|$ and $C_{i, k}$ is the processing capacity

\footnotetext{
${ }^{5}$ Chandy [12] has shown that the last-in-first-out with preemptive resume (LIFO-PR) discipline possesses the properties similar to PS.
}

of node $i$ when there are $k$ customers at the node. Note that in this generalized queueing network model, the Poisson arrival can depend on the total population, whereas the service rate is a function of its local queue size. This fact is reflected in the expressions (61) and (62), which can be viewed as generalizations of the numerator and denominator of (44), respectively. The parameters $\left\{e_{i, r}\right\}$ are similar in interpretation to the parameters $\left\{\Gamma_{i}\right\}$ of (35) and satisfy the system.

$$
e_{j, s}=\theta_{0, j, s}+\sum_{i, r} e_{i, r} \theta_{i, r, j, s}
$$

where $\theta_{0, j, s}$ is the probability that an externally arriving customer enters at node $j$ and is a class-s customer. Hence $\lambda_{n} \theta_{0, j, s}$ represents the (Poisson) external rate of class-s customers at station $j$ when the total population of the network is $n . p_{0}$ of (59) is the probability that the entire system is empty and is uniquely determined by the normalization condition $\Sigma_{\boldsymbol{K}} p_{\boldsymbol{K}}=1$.

If we combine the different classes of customers, we obtain the joint distribution of

$$
k=\left(k_{1}, k_{2}, \cdots, k_{N}\right)
$$

as the marginal distribution of (59). By virtue of the reproducing property of multiple Poisson distributions, we have

$$
p_{k}=p_{0} \Lambda(|| k||) \prod_{1 \leqslant i \leqslant N} \gamma_{i, k_{i}} W_{i}^{k_{i}}
$$

where

$$
W_{i}=\sum_{1 \leqslant r \leqslant R} e_{i, r} E\left\{W_{i, r}\right\}
$$

is the expected total work that a customer requires of node $i$ during the customer's entire life. If, in particular, the arrival rate is a constant $\lambda$, then we have

$$
p_{k}=\prod_{1 \leqslant i \leqslant N} p_{i, k_{i}}
$$

where $p_{i, k_{i}}$ is the marginal distribution for node $i$

$$
p_{i, k_{i}}=p_{i, 0} \beta_{i, k_{i}}\left(E\left\{W_{i}\right\}\right)^{k_{i}}
$$

The parameters $\left\{\beta_{i, k_{i}}\right\}$ of (67) are similar to the $\left\{\beta_{k}\right\}$ of (44)

$$
\beta_{i, k}=\lambda^{k} \gamma_{i, k}=\lambda^{k} /\left(C_{i, 1} \times C_{i, 2} \times \cdots \times C_{i, k}\right) .
$$

\section{Generalized Routing Behavior}

We have assumed up to now that customer's routing within a network is governed by a first-order Markov chain $\left\{\theta_{i, j}\right\}$ or $\left\{\theta_{i, r, j, s}\right\}$. That is, when a customer (of class-r) completes his service at node $i$, he immediately joins the queue at node $j$ (as a class-s customer) or leaves the system. Recently Kobayashi and Reiser [88] have shown that such a restriction on the routing behavior can be eliminated entirely and yet the queue distributions $\left\{p_{\boldsymbol{k}}\right\}$ and $\left\{p_{\mathbf{K}}\right\}$ essentially remain the same. In other words, the solutions obtained earlier are completely robust with respect to routing behavior. 
The derivation of this result can be outlined as follows: consider a class of routing transition characteristics representable by a $h$ th-order Markov chain (where $h$ can be arbitrarily large). Thus the probability that a customer at node $i$ will move upon completion of service to node $j$ depends not only upon (his present location) $i$, but also upon his past history, say the previous $(h-1)$ transitions. We then define the state of a customer by an $h$-tuple

$$
s=\left(i_{1}, i_{2}, \cdots, i_{h}\right)
$$

The values that $s$ can take are the $N^{h} h$-tuples

$$
(0,0, \cdots, 0),(0,0, \cdots, 0,1), \cdots,(N-1, N-1, \cdots, N-1)
$$

which we will represent for notational convenience by the integers $0,1, \cdots, N^{h}-1$. An appropriate representation of the network is then given by the random process of countably infinite dimension

$$
\mathrm{Y}=\left\{Y_{t}=\left(Y_{s, \tau}\right)_{t}:\left(0 \leqslant s<N^{h}, \tau=0,1, \cdots\right), 0 \leqslant t<\infty\right\} .
$$

The process $Y$, like the process $X$ of (57), is not itself a Markov process, but is reducible from a Markov process defined over an appropriately augmented state space. Note that in our integer representation of the state $s$, the server of a customer in state $s$ is uniquely determined by the relationship

$$
i=s(\operatorname{modulo} N) \text {. }
$$

Then the steady-state probability that the process $Y$ takes on the value

$$
K=\left\{\left(k_{s, \tau}\right): 0 \leqslant s<N^{h}, \tau=0,1, \cdots\right\}
$$

is given by following expression

$$
\begin{aligned}
p_{K}= & \lim _{t \rightarrow \infty} \operatorname{Pr}\left\{Y_{t}=K\right\} \\
= & p_{0} \Lambda(|| K||) \prod_{1 \leqslant i \leqslant N} \gamma_{i, k_{i}} \prod_{s: s=i(\bmod N)} \prod_{0 \leqslant \tau<\infty} \\
& \cdot\left(e_{s, \tau} E\left\{W_{s, \tau}\right\}\right)^{k} s, \tau / k_{s, \tau} !
\end{aligned}
$$

where

$$
k_{i}=\sum_{s: s=i(\bmod N)} \sum_{0 \leqslant \tau<\infty} k_{s, \tau} .
$$

The interpretation of the parameters in (74) should be selfevident.

Again by use of the self-reproducing property of the Poisson distribution, the distribution of

$$
k=\left(k_{1}, k_{2}, \cdots, k_{n}\right)
$$

is given by the formula (65). The parameter $W_{i}$ in that expression is now defined by

$$
W_{i}=\sum_{s: s=i(\bmod N)} \sum_{0 \leqslant \tau<\infty} e_{s, \tau} E\left\{W_{s, \tau}\right\}
$$

This quantity is again the total amount of work that a customer will require of server (station) $i$ during his entire life within the network.

One final remark: the notion of customer state $s$ as defined by $(70)$ can be further extended to allow for multiclass customers. For this extension we define $s$ by

$$
s=\left(\left\langle i_{j}, r_{j}\right\rangle: 1 \leqslant j \leqslant k\right)
$$

\section{DISCRETE-TIME QUEUEING SYSTEMS AND THEIR APPLICATIONS}

We have thus far dealt with continuous-time queueing systems: both interarrival time and service time are considered to be nonnegative real-valued random variables. We should recognize, however, that many actual systems we encounter operate on a discrete-time basis. That is, we discern a natural elementary unit of time in a given system. Examples are the machine cycle time of a processor, the bit or byte duration of signals on a channel or transmission line. For practical purposes it is usually justifiable to approximate such system operations in terms of continuous-time models, because of the elegance and richness of mathematical techniques for continuous-time models. In certain problems, however, continuous-time approximations are not applicable and hence we must formulate them as pure discrete-time problems. Typical examples of our interest are queueing problems associated with synchronous communication systems, including the conventional STDM and packet-switching systems with time-slotting, e.g., a slotted ALOHA multiplexing scheme [1]. In such systems, all events are allowed to occur only at definite regularly spaced time points.

Aside from such discrete structures of intrinsic nature as cited above, we often find it computationally convenient and efficient to deal with discrete time systems when we solve a given problem by digital computations. Neuts and his associates [61], [111], [112] have investigated the numerical aspects of solving various types of single-server queueing models.

One might quickly think that every result and formula derived for a continuous-time model can be translated into its discrete-time analog in an obvious fashion. Unfortunately, this does not always turn out to be the case, as discussed below. The major factor that hampers our generalizing the results of Markovian queueing networks, for example, seems to be the fact that a Poisson process possesses two different discretetime analogs: one is a Bernoulli sequence, the other a Poisson sequence as will be defined and discussed in the sequel. We will also find it necessary to define the notion of processor sharing (PS) different from the round-robin (RR) scheduling, although PS was initially introduced as the limiting case of RR.

\section{Discrete Analog of the System $M / M / 1$}

Let us assume that the time axis is segmented into a continguous sequence of time intervals (slots) of duration $\Delta$. In an ordinary data transmission/processing system, $\Delta$ may cor- 
respond to the pulse duration, byte length, packet size, or to any data unit of fixed size. If we assume that no more than one customer or unit may arrive in a given slot, and the arrivals in different time slots are statistically independent, then the arrival sequence may be identified with a sequence of 0 's and 1's, $\mathbf{x}=\left(X_{1}, X_{2}, \cdots\right) ; X_{k}=1$ if a customer arrives in the $k$ th slot and 0 otherwise. The random variables $X=\left\{X_{k}\right\}$ are independent and identically distributed, $X_{k}=0$ or 1 , and $\operatorname{Pr}\left\{X_{k}=1\right\}=\lambda$ with $0 \leqslant \lambda \leqslant 1$. We say that $X$ is a Bernoulli sequence and that $X_{k}$ is the outcome on the $k$ th trial. The total count of arrivals $N_{m \Delta}$ in the first $m$ slots has the binomial distribution (with parameter $\lambda$ )

$$
\operatorname{Pr}\left\{N_{(m+i) \Delta}-N_{i \Delta}=n\right\}=C(m, n) \lambda^{n}(1-\lambda)^{m-n}
$$

where $C(m, n)$ is the binomial coefficient

$$
C(m, n)=\frac{m !}{n !(m-n) !} .
$$

Here $\mathbf{N}^{d}=\left\{N_{i \Delta}: 0 \leqslant i<\infty\right\}$ is a discrete counting processthe counterpart of the continuous-time counting process defined in Section II-and $N_{i \Delta}$ is the number of arrivals in the first $i$ slots. For a Poisson process, the interarrival times $\left\{\tau_{i}\right\}$ were iid and exponentially distributed; for the process $\mathbf{N}^{d}$ they are iid with the geometric distribution

$$
f_{k}=\operatorname{Pr}\left\{\tau_{i}=k \Delta\right\}=\lambda(1-\lambda)^{k-1}, \quad 1 \leqslant k<\infty .
$$

We also assume that the service times $\left\{S_{i}\right\}$ are iid with

$$
g_{k}=\operatorname{Pr}\left\{S_{i}=k \Delta\right\}=\mu(1-\mu)^{k-1}, \quad 1 \leqslant k<\infty
$$

The geometric distribution, like the exponential distribution, enjoys the memoryless property; the conditional probability that $\tau_{i}$ is larger than $(k+j) \Delta$ given (the condition) that it is already larger than $j \Delta$ is independent of the value of $j$,

$$
\operatorname{Pr}\left\{\tau_{i}>(k+j) \Delta \mid \tau_{i}>j \Delta\right\}=\operatorname{Pr}\left\{\tau_{i}>k \Delta\right\} .
$$

Thus if the state of the system is described by the random process $\mathrm{X}=\left\{X_{j}: 0 \leqslant j<\infty\right\}$ where $X_{j}$ is the number of customers waiting or in service at time $j \Delta$, then $\mathbf{X}$ is a Markov process (chain). If $\rho=\lambda / \mu<1$, the process $X$ is recurrent and a stationary distribution for the queue length exists and is given by the same formula as in the continuous case. The departure process is similarly a Bernoulli sequence when the initial distribution is appropriately chosen. Furthermore, the notion of queue-dependent arrival and processing rates as discussed in Section III is equally applicable here.

\section{Discrete Analog of the System $M / G / 1$}

The $M / G / 1$ system provides another case in which the discrete-time interpretation of results in its continuous-time model is fairly straightforward. The system $M / G / 1$ (Poisson arrival/general service time/single server) is discussed in most of the standard references on queueing theory (Cohen [26], Feller [39], Kleinrock [79]) and hence will not be reviewed further here. Meisling [107] discusses the discrete-time $M / G / 1$ system.
We assume that arrivals are characterized by a Bernoulli sequence and that the queue discipline is any nonpreemptive work-conserving discipline. Nenpreemptive means that once a customer enters service, he must be processed until completion before the server selects the next customer for service. Then the method of imbedded Markov chains developed by Kendall [69] for the continuous-time model is applicable here also.

Let $Y_{i}$ be the number of customers in the system just after the service completion of the $i$ th customer and let $Z_{j}$ be the number of customers entering the system during the service of that job. Then the sequences $\left\{Y_{i}\right\}$ and $\left\{Z_{i}\right\}$ are related by the recurrence relation

$$
Y_{i+1}=Y_{i}-\chi_{\left(Y_{i}>0\right)}+Z_{i+1}
$$

The probability generating function of the stationary distribution $\left\{\pi_{k}\right\}$ of $Y_{i}$

$$
\Pi(z)=\sum_{0 \leqslant k<\infty} \pi_{k} z^{k}=\lim _{i \rightarrow \infty} E\left\{z^{Y_{i}}\right\}
$$

must satisfy the equation

$$
\Pi(z)=\lim _{i \rightarrow \infty} E\left\{z^{Y_{i+1}}\right\}=\lim _{i \rightarrow \infty} E\left\{z^{Z_{i+1}}\right\} E\left\{z^{Y_{i}-\chi\left(Y_{i}>0\right)}\right\} .
$$

In (84) we have used the independence of $Y_{i}$ and $Z_{i}$. If we define $B(z)$ to be the probability generating function of the number of arrivals during a service period of a customer, we have

$$
\begin{aligned}
B(z) & =\sum_{0 \leqslant n<\infty} z^{n} \sum_{n \leqslant j<\infty} g_{j} C(j, n) \lambda^{n}(1-\lambda)^{j-n} \\
& =G(1-\lambda+\lambda z)
\end{aligned}
$$

where

$$
G(z)=\sum_{0 \leqslant j<\infty} g_{j} z^{j}
$$

is the generating function of the service time distribution $\left\{g_{j}\right\}$. Equations (84) and (85) readily show that

$$
\Pi(z)=B(z)[\Pi(0)+(1 / z)(\Pi(z)-\Pi(0))]
$$

or equivalently

$$
\Pi(z)=\Pi(0) B(z)(z-1) /(z-B(z)) .
$$

The solution given in (87) contains the undetermined parameter $\Pi(0)$-the value of the function $\Pi(z)$ when $z=0$. This number is fixed by the normalization condition $\Pi(z)=1$ when $z=1$. Allowing $z \rightarrow 1$ in (87) and noting that $B(1)=1$ we have $\left(1-\left.(d / d z) B(z)\right|_{z=1}\right)=\Pi(0)$. If we set $\rho=1-\Pi(0)$, then

$$
\rho=\left.(d / d z) R(z)\right|_{z=1}=\lambda E\left\{S_{i}\right\}
$$

while the parameter $\rho$ is the server utilization as before. It is 
well known for a continuous-time $M / G / 1$ system that the distribution $\left\{\pi_{k}\right\}$ is the same as the distribution $\left\{p_{k}\right\}$ of the number of customers observed at a randomly chosen instant of time and hence equals the time-average distribution as well. This important property holds for a discrete-time $M / G / 1$ system. One proof of this fact makes use of the notion of supplementary variables [31]. Thus the probability generating function of the sequence $\left\{p_{k}\right\}$ is given by

$$
P(z)=(1-\rho) B(z)(z-B(z))
$$

Schmookler [135] discusses an extension of the result (89) to the case of compound or bulk arrivals. In such a system we allow more than one customer to enter the system at a time. We denote this system by the notation $M^{(A)} / G / 1$ where the $A$ of the superscript is the variable representing the number of customers in an arriving group. Let $\lambda$ represent the group arrival rate and suppose that the size of the $n$th group is a random variable $A_{n}, \operatorname{Pr}\left\{A_{n}=k\right\}=a_{k}$, with

$$
\Phi(z)=\sum_{0 \leqslant k<\infty} a_{k} z^{k} .
$$

Note that the arrival process is a compound process. It is a discrete generalization of the compound Poisson process discussed earlier in Section II. Define a set of parameters $\left\{\lambda_{n}\right\}$ by

$$
\lambda_{n}= \begin{cases}1-\lambda, & \text { if } n=0 \\ a_{n} \lambda, & \text { if } 1 \leqslant n<\infty .\end{cases}
$$

Then $\lambda_{n}$ may be interpreted as the arrival rate of a group of size $n$. We then define the generating function $\Lambda(z)$ by

$$
\Lambda(z) \doteq \sum_{0 \leqslant n<\infty} \lambda_{n} z^{n}=1-\lambda+\lambda \Phi(z) .
$$

The (left) composition of the function $G$ with respect to the function $\Lambda$, which we denote by $B^{(A)}$, is

$$
B^{(A)}(z)=(G \cdot \Lambda)(z)=G(\Lambda(z))=G(1-\lambda+\lambda \Phi(z))
$$

is the probability generating function of the total number of customers arriving during a service period. Formula (87) may then be generalized to the $M^{(A)} / G / 1$ system yielding

$$
\Pi^{(A)}(z)=\Pi^{(A)}(0) B^{(A)}(z)(z-1) /\left(\dot{z}-B^{(A)}(z)\right) .
$$

The constant $\Pi^{(A)}(0)$ is now given by

$$
\Pi^{(A)}(0)=(1-\rho) /\left.(d / d z) \Phi(z)\right|_{z=1}=(1-\rho)
$$

where $\rho=\lambda E\{S\} E\{A\}$ and $E\{A\}$ is the expected size of a group. Similarly (89) is also generalized to

$$
P^{(A)}(z)=(1-\rho) B^{(A)}(z)(z-1) /\left(z-B^{(A)}(z)\right) .
$$

Computation of the queue distribution $\left\{p_{k}\right\}$ can, in principle, be obtained by inverting the generating functions in equations (89) or (96). In practice, a simple recurrence relation for the $\left\{p_{k}\right\}$ may be derived from (89) or (96) provided $B(z), B^{(A)}(z)$, and $\Phi(z)$ are rational functions of $z$. See Kendall [96] and Meisling [107] for representations of this type. The average queue size and response time are easily found from (89) or (96), together with Little's formula.

If we assume further that the queue discipline is FIFO, the waiting and response time distributions can be obtained also in terms of the generating functions (as contrasted with LaplaceStieltjes transform solutions obtained for the continuous-time $M / G / 1$ ). For other scheduling disciplines (LIFO, random) it seems not too difficult to extend known results for the continuous-time case. The case of finite capacity (waiting room) is an important one for the analysis of buffer storage and the model can be treated using techniques similar to those developed by Riordan [129] and Keilson [68]. Schmookler [135] discusses this problem in detail. See also Neuts [111] for related subjects.

\section{Processor-Sharing (PS) in a Discrete-Time-System and Applications}

In Section IV we saw that under the PS discipline [76], the system $M / G / 1$ behaves as if it were an $M / M / 1$ system; hence the departure process exhibits a Markovian property, namely, a Poisson process with the same rate as the input. A proper translation of this interesting property for a discrete-time system requires the following definition.

Definition: PS in a discrete-time queueing system is the scheduling rule which selects, in every time slot, one of the $n$ customers in the system randomly and equally likely and then processes this customer for one slot time.

Thus if the service requirement of a customer is $k \Delta$, then it must be scheduled and processed $k$ times before its service is completed. Note also that the so-called round-robin (RR) scheduling is different from the PS discipline defined above, although in the limit as $\Delta \rightarrow 0$ both RR and PS converge to the same continuous-time PS discipline originally defined by Kleinrock [76] .

Kobayashi [85] has proved formally that for the discretetime PS discipline defined here all of the results obtained for the continuous-time model can be carried over directly including those relating to queue-dependent arrival and processing rates and different classes of customers with arbitrary service distributions.

It is worthwhile to cite a number of possible applications of the notion of PS. The first example relates to the congestion analysis of store-and-forward packet-switching computer systems. A message consists of one or more packets; individual packets are usually processed and transferred as independent units. Thus if we view a transmission link as a service facility, it is essentially an $M / G / 1$ system with the server operating under the PS queue discipline when the message flows between a given pair of nodes can be characterized by a Poisson process.

The second example arises from modeling the ALOHA multiplexing scheme [87]. In this scheme a user transmits his data messages into a random-access channel in the form of packets. If a packet occupies the channel for the entire packet duration, it will be successfully transmitted to its destination. 
If, on the other hand, more than one user transmits packets simultaneously and these transmission times overlap, even partially, then all of the packets involved are considered lost, and they must be retransmitted after some randomly chosen delays. If we choose the retransmission delay of any outstanding packet as a discrete-time random variable with the geometric distribution

$$
\begin{aligned}
\operatorname{Pr}\{\text { retransmission delay }=d \Delta\}=(1-r) r^{d-1}, & \\
1 & \leqslant d<\infty
\end{aligned}
$$

for some $r, 0<r<1$, then at a given time slot, all of the outstanding packets will attempt retransmissions independently, and with equal probability $r$. The rate of successful transmission depends, of course, on the level of contention: the larger the number of outstanding packets in the system, the lower the channel throughput. Thus the ALOHA channel (which we define as the combination of the random-access channel and retransmission devices of the individual user terminals) can be appropriately modeled as a processor-sharing system (or more aptly we should call it a capacity-sharing channel with queuesize dependent processing rate (or capacity). For further discussion of this application the reader is referred to Kobayashi, Onozato, and Huynh [87]. A slightly different model for satellite communication is discussed in Gavish and Konheim [47]. The reader is also directed to Carleial and Hellman [11] and Kleinrock and Lam [81] for the performance analysis of the slotted ALOHA system.

\section{Discrete Analog of the System $M / G / \infty$}

In this case an analogy between a continuous-time model and a discrete-time one is essentially of a different nature from those discussed previously. Consider a Bernoulli arrival process at a station with infinitely many parallel servers. Because the service times of individual customers are independent, it is possible that more than one server can complete the service of a customer in a given time slot, except for the special case when all customers require exactly the same amount of service, that is, in the system $M / D / \infty$ (where $D$ stands for deterministic). Thus it is clear that the beautiful property-Bernoulli input $\rightarrow$ Bernoulli output-cannot hold for the discrete system $M / G / \infty$. This failure, in fact, creates a major difficulty, when we attempt to model a network of queues of discrete-time structure. As we discussed earlier the $M / G / \infty$ system possesses several interpretations and useful applications: it is a mathematical representation of random delay. It represents, in a closed Markovian network with finite population, the population source itself (such as a set of terminals).

Consider a compound arrival process in which the $\left\{\lambda_{n}\right\}$ of (91) arise from a Poisson distribution

$$
\lambda_{n}=\lambda_{n}=\lambda^{n} e^{-\lambda / n !}, \quad 0 \leqslant n<\infty .
$$

This arrival process is a more natural one than the Bernoulli sequence as a discrete-time counterpart of a Poisson process. Start with a Poisson process of rate $\mu$. The number of events (arrivals) in a finite interval of length $\Delta$ has a (Poisson) distribution with expectation $\lambda=\mu \Delta$. We call the discretized Poisson process, $\mathbf{N}^{d}=\left\{N_{i \Delta}: 0 \leqslant i<\infty\right\}$-obtained by only observing the continuous-time Poisson process $\mathbf{N}=\left\{N_{t}: 0 \leqslant t<\infty\right\}$ at the discrete set of time points $i \Delta-\mathrm{a}$ Poisson sequence; $N_{i \Delta}$ has the distribution

$$
\operatorname{Pr}\left\{N_{i \Delta}=k\right\}=(i \mu \Delta)^{k} e^{-i \mu \Delta} / k !, \quad 0 \leqslant k<\infty .
$$

and the generating function

$$
\Lambda(z)=e^{\lambda(z-1)} .
$$

With this assumption made concerning the arrival process, most of the results obtained for the time-continuous $M / G / \infty$ system are translated into the discrete-time case. Let $\left\{g_{k}\right\}$ denote, as before, the probabilities that the service time (or delay) in the system $M / G / \infty$ are $k \Delta(0 \leqslant k<\infty)$. Assuming that the system is initially empty, we have the following expression for the probability that there are $k$ customers in the system during the $r$ th time unit $(1 \leqslant r<\infty)$;

$$
p_{k}(r)=\left[\lambda \sum_{0 \leqslant i<r} G_{i}{ }^{c}\right]^{k} \exp \left[-\lambda \sum_{0 \leqslant i<r} G_{i}{ }^{c}\right] / k !
$$

where $\left\{G_{i}^{c}\right\}$ is the complement of the cumulative distribution of the $\left\{g_{k}\right\}$,

$$
G_{i}^{c}=\operatorname{Pr}\{S>i \Delta\}=\sum_{k>i} g_{k} .
$$

In the limit as $r \rightarrow \infty$, we therefore have the following stationary state distribution:

$$
p_{k}=\lim _{r \rightarrow \infty} p_{k}(r)=\rho^{k} e^{-\rho} / k !
$$

in which $\rho$ represents the average number of servers busy (or the average number of customers in the system) at a given time.

$$
\rho=\lambda \sum_{0 \leqslant i<\infty} G_{i}^{c}=\lambda E\{S\} / \Delta
$$

We can show that the number of departures in different time slots are independent and the number of departures at the end of the $r$ th time slot has a Poisson distribution with parameter

$$
\lambda \sum_{0 \leqslant i \leqslant r} g_{i}
$$

Hence, in the limit as $r \rightarrow \infty$, the output process is a homogeneous Poisson sequence with rate $\lambda$.

An attempt to generalize the results of queue-dependent arrival and/or processing-rates has thus far turned out to be unsuccessful. Thus the formulas (43)-(45) do not hold in a discrete-time model, whereas formulas (46)-(52) directly carry over to the discrete-time case. For detailed discussions on the subject of the present section, the reader is referred to Kobayashi [85]. 


\section{Discrete-Time Analog of the System GI/G/1}

In the discrete-time $M / G / 1$ system discussed earlier, the interarrival times $\left\{\tau_{i}\right\}$ were iid with a geometric distribution. If we remove the hypothesis as to the distributional form, and allow an arbitrary distribution $\left\{f_{k}\right\}$,

$$
\operatorname{Pr}\left\{\tau_{i}=k \Delta\right\}=f_{k},
$$

the resulting system is a discrete-time system $G I / G / 1$ (general independent arrivals/general service time/single server). Lindley, in a fundamental paper [101], showed how the stationary waiting time distribution, $\lim _{n \rightarrow \infty} \operatorname{Pr}\left\{W_{n} \leqslant x\right\}$ ( $W_{n}=$ waiting time of the $n$th customer), was the solution of an integral equation. In general the solution involves techniques from complex function theory. Under the assumption that the distributions $\left\{f_{k}\right\}$ and $\left\{g_{k}\right\}$ are of compact support $\left(f_{k}=g_{k}=0\right.$ for $k>K$ ), Konheim [95] gives a simple method for calculating the waiting time distribution of the $n$th customer as $n \rightarrow \infty$

$$
h_{k}=\lim _{n \rightarrow \infty} \operatorname{Pr}\left\{W_{n}=k \Delta\right\}
$$

where $W_{n}$ is the waiting time of the $n$th customer. The waiting times $\left\{W_{n}\right\}$ satisfy the recurrence equation

$$
W_{n+1}=\left(W_{n}+S_{n}-\tau_{n+1}\right)^{+}, \quad 1 \leqslant n<\infty ; W_{1}=0
$$

where $a^{+}=\max (a, 0)$. Let

$$
\begin{aligned}
& F(z)=\sum_{0 \leqslant k<\infty} f_{k} z^{k} \\
& G(z)=\sum_{0 \leqslant k<\infty} g_{k} z^{k} \\
& H(z)=\sum_{0 \leqslant k<\infty} h_{k} z^{k} .
\end{aligned}
$$

From the hypothesis that $\left\{f_{k}\right\}$ and $\left\{g_{k}\right\}$ have compact support, it follows that $F$ and $G$ are polynomials. Form the rational function

$$
S(z)=[1-F(1 / z) G(z)] /(1-z)
$$

and factor $S(z), S(z)=S^{+}(z) S^{-}(z)$ with

$$
\begin{array}{ll}
S^{+}(z)=C_{1} \prod\left(z-v_{i}^{+}\right)^{r i}, & 1<\left|v_{i}^{+}\right| \\
S^{-}(z)=C_{2} z^{-C_{3}} \prod\left(z-\nu_{i}^{-}\right)^{S i}, & 0<\left|v_{i}^{-}\right|<1
\end{array}
$$

choosing the constants $C_{1}$ and $C_{2}$ so that $S^{+}(1)=1$. Equations (112), (113) provide a Wiener-Hopf-like factorization of $S(z) ; 1 / S^{+}(z)$ is analytic in the open unit disk $\{z:|z|<1\}$ while $1 / S^{-}(z)$ contains the singularities of $S(z)$ in this disk. It can be shown that $H(z)=1 / S^{+}(z)$ so that the determination of the stationary waiting time distribution requires just (!) the ability to factor polynomials. The assumption that $F$ and $G$ are polynomials is of course unnecessary. The general case may be obtained by approximating an actual distribution by one of finite support. This method should be compared with various techniques for the solution of the $G I / G / 1$ system (Pollaczek [124], Spitzer [141], and Cohen [26]) all of which use complex analytic methods.

\section{Buffer Storage Analysis}

One of the applications of the discrete-time model is in the study of buffer storage. The main analytic problem is the determination of the buffer overflow probability. A common feature found in many computer-communication or terminaloriented systems is that message generation is usually of a busty nature. Thus the multiplexing of a transmission link or channel is commonly adopted to enhance the utilization of the line. Then some type of buffer storage is necessary to transform the arriving stream with large variation in time into an outgoing flow of a more regular nature. The buffer space should be sufficient to accommodate a large queue of messages which may occasionally develop. Fig. 4 illustrates that the buffer storage problem is essentially a queueing system which is provided with only a finite capacity to hold waiting customers.

The idea of multplexing is applied not only to transmission media but also to processing. For example, a processor is shared by a number of teletype terminals, or by a set of other processors connected via a network. Quite often the flow of data from the individual lines is low compared to the processing capability of the host computer, either due to the slow message generation rate or to the limited bandwidth of the transmission facility. In such circumstances a high utilization of the processor can be achieved only by means of multiplexing of some kind. Buffer storage is then required to perform such a multiplexing operation. The buffer's capacity and storage allocation strategy is of great importance for the cost-effective design of a computer-communication system. Analyses of various schemes are discussed by Gaver and Lewis [45], Chang [15], and Schultz [136].

In the remainder of this section we focus on a queueing model pertinent to such studies. We should call to the user's attention the work in [19], [24], [25], [44], [50], [63], [64], [98], [118] on related subjects. Here we follow primarily the formulation and results reported by Wyner [151] .

Let us consider a discrete-time system in which $\alpha_{k}$ data units arrive in the $k$ th slot for transmission (or processing). During the same interval $\beta_{k}$ data units will be sent (or processed). We assume that the two-dimensional process $\left\{\left(\alpha_{k}\right.\right.$, $\beta_{k}$ ): $\left.1 \leqslant k<\infty\right\}$ of births (the $\left\{\alpha_{k}\right\}$ ) and deaths (the $\left\{\beta_{k}\right\}$ ) is such that the differences $\left\{\gamma_{k}\right\}$ defined by

$$
\gamma_{k}=\alpha_{k}-\beta_{k}, \quad 1 \leqslant k<\infty
$$

satisfy the following properties: 1) the $\left\{\gamma_{k}\right\}$ are iid random variables; 2) $E\left\{\gamma_{k}\right\} \leqslant 0$; and 3) $\operatorname{Pr}\left\{\gamma_{k}>0\right\}>0$. Because births will occasionally exceed deaths, at least some of the time [property 3)], the system will not be able to handle all of the available data. Therefore, buffer storage (or a waiting room) must be interposed between the arriving source and the 


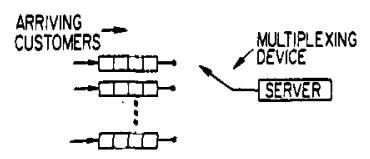

Fig. 4. Buffer storage.

server. Let $L$ [data units] be the size of the buffer capacity. Occasionally the buffer will be completely full, and at these times the excess $\gamma_{k}$ of arrival over service will be lost. We call this event a buffer overflow.

Our main objective here is to find out how the behavior of the buffer is characterized in terms of the random process $\left\{\left(\alpha_{k}, \beta_{k}\right)\right\}$ and the buffer capacity $L$. Because of property 1$)$, we can define the state of the system of buffer storage at time $k$ as the queue length $X_{k}$ [data units], where

$$
X_{0}=0
$$

and for $1 \leqslant k<\infty$,

$$
X_{k}= \begin{cases}0, & \text { if } X_{k-1}+\gamma_{k}<0 \\ X_{k-1}+\gamma_{k}, & \text { if } 0<X_{k-1}+\gamma_{k} \leqslant L \\ L, & \text { if } X_{k-1}+\gamma_{k}>L\end{cases}
$$

or more concisely

$$
\begin{aligned}
X_{k} & =\min \left\{\max \left[X_{k-1}+\gamma_{k}, 0\right], L\right\} \\
& =L-\left\{L-\left(X_{k-1}+\gamma_{k}\right)^{+}\right\}^{+} .
\end{aligned}
$$

The recurrence equation [(116) or (117)] is similar to (82) for the $M / G / 1$ system. Equation (116) bears an even stronger resemblance to the recurrence equation (107) for the waiting times of customers in the $G I / G / 1$ queueing system. In fact, in the limit as $L \rightarrow \infty$ (116) reduces exactly to (107). This correspondence holds if we interpret $\alpha_{k}$ as the service time of the $k$ th customer, $\beta_{k}$ as the interarrival time between the $k$ th and $(k+1)$ st customers, and $X_{k}$ as the waiting time of the $(k+1)$ st customer. Note that in both interpretations (the buffer storage system and the $G I / G / 1$ system) $X_{k}$ represents an unfinished amount of work to be found in the system by the next arriving customer. Note also, that in our formulation the service or scheduling discipline can be any work-conserving one: it is not necessary to assume that the processor or channel processes data in the buffer storage in the order of their arrival. When the service discipline is FIFO, then $X_{k}$ equals the waiting time of the $(k+1)$ st customer in the analogous queueing system.

Let $p^{(L)}(n)$ denote the probability that the buffer state (the number of data units in the system) is $n$ [data units] after the system of capacity $L$ [data units] reaches its equilibrium.

$$
p^{(L)}(n)=\lim _{k \rightarrow \infty} \operatorname{Pr}\left\{X_{k}=n\right\} .
$$

Because of the analogy observed above, we see that in the limit as $L \rightarrow \infty$, we can use the various results established for the system $G I / G / 1$. Of particular interest and importance to us is the asymptotic behavior of $P^{(L)}(n)$ for large $L$ and $n$. As we will demonstrate below, the results and techniques discussed by Kingman [72] , [73], Kobayashi [84], Ross [131], and Wyner
[151] for bounds of the waiting time distribution are applicable in the present problem.

For a continuous-time $G I / G / 1$ system (in which the $\left\{\alpha_{k}\right\}$ and $\left\{\beta_{k}\right\}$ and hence the $\left\{\gamma_{k}\right\}$ as well are real-valued random variables) the following inequality was derived by Kingman [73]

$$
a e^{-\vartheta_{0} t} \leqslant \lim _{k \rightarrow \infty} \operatorname{Pr}\left\{X_{k}>t\right\} \leqslant e^{-\vartheta_{0} t}
$$

where

$$
\begin{aligned}
a & =1 /\left[\sup _{t>0} E\left\{e^{\vartheta_{0}(\gamma-t)} \mid \gamma \geqslant t\right\}\right] \\
& =\inf _{t>0} \int_{t}^{\infty} d H(y) / \int_{t}^{\infty} e^{\vartheta_{0}(y-t)} d H(y) .
\end{aligned}
$$

Here the function $H(t)$ is the distribution function of the variable $\gamma_{k}$ and $\vartheta_{0}$ is the unique positive real root of the equation

$$
E\left\{\exp \left(\vartheta \gamma_{k}\right)\right\}=\int_{-\infty}^{\infty} e^{\vartheta t} d H(t)=1 .
$$

The right-hand side of the inequality (119) was proved by using Kolmogorov's inequality for martingales. Kobayashi [84] derived a similar expression for the transient distribution applying the same Kolmogorov's inequality to submartingales. The derivation is quite analogous to that of the wellknown Chernoff bound which has been applied to bound the error probability in statistical decision theory. Ross [131] recently found a tighter upper bound, also based upon the martingale approach.

$$
a e^{-\vartheta_{0} t} \leqslant \lim _{k \rightarrow \infty} \operatorname{Pr}\left\{X_{k}>t\right\} \leqslant b e^{-\vartheta_{0} t}
$$

where

$$
\begin{aligned}
b & =1 /\left[\inf _{t>0} E\left\{e^{\vartheta_{0}(\gamma-t)} \mid \gamma \geqslant t\right\}\right] \\
& =\sup _{t>0} \int_{t}^{\infty} d H(y) / \int_{t}^{\infty} e^{\vartheta} 0^{(y-t)} d H(y) \leqslant 1 .
\end{aligned}
$$

A direct translation of the above result applied to the discretetime system we are considering is as follows:

$$
a z_{0}{ }^{-n} \leqslant \lim _{k \rightarrow \infty} \operatorname{Pr}\left\{X_{k}>n\right\} \leqslant b z_{0}{ }^{-n}
$$

where

$$
\begin{aligned}
a & =1 /\left[\sup _{n \geqslant 0} E\left\{z_{0} \gamma-n \mid \gamma \geqslant n\right\}\right] \\
& =\inf _{n \geqslant 0} \sum_{n \leqslant i<\infty} h_{i} / \sum_{n \leqslant i<\infty} z_{0}{ }^{i-n} h_{i}
\end{aligned}
$$


and

$$
\begin{aligned}
b & =1 /\left[\inf _{n \geqslant 0} E\left\{z_{0}{ }^{\gamma-h} \mid \gamma \geqslant n\right\}\right] \\
& =\sup _{n>0} \sum_{n \leqslant i<\infty} h_{i} / \sum_{n \leqslant i<\infty} z_{0}{ }^{i-n} h_{i} .
\end{aligned}
$$

Here $z_{0}(>1)$ in $(125),(126)$ is the unique positive real root of the equation

$$
H(z)=1
$$

where $H(z)$ is the probability generating function $(\mathrm{pgf})^{6}$ of the integer-valued random variable $\gamma_{k}$ and is given by the product of the pgf's of $\left\{\alpha_{k}\right\}$ and $\left\{\beta_{k}\right\}$.

$$
H(z)=E\left\{z^{\gamma_{k}}\right\}=E\left\{z^{\alpha_{k}}\right\} E\left\{z^{-\beta_{k}}\right\}=F(z) G\left(z^{-1}\right)
$$

Less tight but more computationally available bounds are based upon the probability distribution $\left\{f_{n}\right\}$ of the random variables $\left\{\alpha_{k}\right\}$,

$$
\begin{aligned}
a & \geqslant 1 /\left[\sup _{n \geqslant 0} E\left\{z_{0}^{\alpha-n} \mid \alpha \geqslant n\right\}\right] \\
& =\inf _{n \geqslant 0} \sum_{n \leqslant i<\infty} f_{i} / \sum_{n \leqslant i<\infty} z_{0}{ }^{i-n} f_{i}
\end{aligned}
$$

and

$$
\begin{aligned}
b & \leqslant 1 /\left[\inf _{n \geqslant 0} E\left\{z_{0} \alpha-n \mid \alpha \geqslant n\right\}\right] \\
& =\sup _{n \geqslant 0} \sum_{n \leqslant i<\infty} f_{i} / \sum_{n \leqslant i<\infty} z_{0}{ }^{i-n} f_{i} .
\end{aligned}
$$

For the discrete-time system under discussion, we can rewrite (124), using the notation of (118), as

$$
a z_{0}{ }^{-n} \leqslant \sum_{n+1} p_{m<\infty}^{(\infty)}(m) \leqslant b z_{0}^{-n}
$$

It is easy to establish the following bounding relation between the distribution $\left\{p^{(\infty)}(n)\right\}$ and $p^{(L)}(L)$, the probability that the buffer of capacity $L$ is full:

$$
p^{(L)}(L) \leqslant \sum_{L \leqslant n<\infty} p^{(\infty)}(n)
$$

Hence, together with the upper bound of (131) we have

$$
p^{(L)}(L) \leqslant b z_{0}^{-(L-1)} .
$$

${ }^{6} H$ is the formal power series associated with the sequence $\left\{h_{k}=\right.$ $\operatorname{Pr}\{\gamma=k\}\}$. $H$ is analy tic in some region if the sequence $\left\{h_{k}\right\}$ vanishes for $k>K$ or $k<K$.
Wyner [151] obtained the following results, based upon quite elaborate arguments, which we will not reproduce here.

1) When $E\left\{\gamma_{k}\right\}=0$, there exist finite constants $c_{0}$ and $c_{1}$ such that

$$
\lim \sup _{L \rightarrow \infty} L p^{(L)}(L)=c_{0}
$$

and

$$
\lim \inf _{L \rightarrow \infty} L p^{(L)}(L)=c_{1} .
$$

Hence we have for an arbitrary $L$,

$$
p^{(L)}(L)=C(L) / L
$$

with

$$
c_{0} \leqslant C(L) \leqslant c_{1}
$$

2) When $E\left\{\gamma_{k}\right\}<0$, there exist finite constants $d_{0}, d_{1}$ such that

$$
\lim \inf _{L \rightarrow \infty} z_{0}{ }^{L} p^{(L)}(L)=d_{0}
$$

and

$$
\lim \sup _{L \rightarrow \infty} z_{0}{ }^{L} p^{(L)}(L)=d_{1} .
$$

Hence for arbitrary $L$,

$$
p^{(L)}(L)=D(L) z_{0}-L
$$

with

$$
d_{0} \leqslant D(L) \leqslant d_{1}
$$

Wyner obtained the results (134)-(141) under the hypothesis that the distribution $\left\{h_{n}\right\}$. of the variables $\left\{\gamma_{k}\right\}$ has compact support; that is, $h_{n}=0$ for $|n|>N_{0}$ for some $N_{0}$.

We will illustrate the use of the various formulas presented here in the following two examples.

Example 1: Suppose that the arrival process has a Poisson distribution with mean $\lambda$ [data units]

$$
\begin{aligned}
f_{n} & =\lambda^{n} e^{-\lambda / n !} \quad 0 \leqslant n<\infty \\
F(z) & =e^{\lambda(z-1)} .
\end{aligned}
$$

Assume as before that the channel sends out data units at a constant rate $\mu$ [data units]

$$
\begin{aligned}
& g_{n}= \begin{cases}1, & \text { if } n=\mu \\
0, & \text { otherwise. }\end{cases} \\
& G(z)=z^{\mu} .
\end{aligned}
$$

Then the characteristic equation is given by

$$
H(z)=z^{-\mu} e^{\lambda(z-1)}=1 .
$$

For $z$ real, (142) can be written in the form 


$$
(z / C)=\exp [(C \lambda / \mu) z / C]
$$

with $C=\exp -\lambda / \mu$. It is known [150] that (143) has the solution

$$
z_{0}=e^{-\lambda / \mu} \sum_{0 \leqslant n<\infty}(n+1)^{n-1}\left[\lambda / \mu e^{-\lambda / \mu}\right] / n !
$$

provided that $\left[\lambda / \mu e^{-\lambda / \mu}\right]<e^{-1}$. This condition holds whenever $\lambda<\mu$.

Example 2: Suppose that the arrival per unit time is characterized by the geometric distribution

$$
f_{n}=(1-r) r^{n} \quad 0<r 1,0 \leqslant n<\infty
$$

with pgf

$$
F(z)=(1-r) /(1-r z)
$$

and expected interarrival time

$$
E\left\{\alpha_{k}\right\}=\left.(d / d z) F(z)\right|_{z=1}=r /(1-r) \text { [data units]. }
$$

Assume that the channel sends out data at a constant rate $\mu$ [data units/unit time], where we assume for simplicity that $\mu$ is an integer:

$$
g_{n}= \begin{cases}1, & \text { if } n=\mu \\ 0, & \text { otherwise }\end{cases}
$$

with pgf $G(z)=z^{\mu}$.

The characteristic equation (127) is given by

$$
H(z)=(1-r)^{-\mu} /(1-r z)=1
$$

or equivalently

$$
r z^{\mu+1}-z^{\mu}+(1-r)=0
$$

For general $r,(145)$ cannot be solved in closed form; numerically it presents no problem and $z_{0}$ may be determined by any of a number of root-finding methods. It is not difficult to show that there exists a unique $z_{0}$ such that $1<z_{0}<1 / r$. The distribution of the variable $\gamma_{k}$ is given by inverting $H(z)$,

$$
h_{i}= \begin{cases}(1-r) r^{i+\mu}, & \text { if } i \geqslant-\mu \\ 0, & \text { if } i<-\mu .\end{cases}
$$

In this case

$$
\sum_{n \leqslant i<\infty} h_{i} / \sum_{n \leqslant i<\infty} z_{0}{ }^{i-n} h_{i}=\left(1-r z_{0}\right) /(1-r)
$$

independent of $n \geqslant 0$. Hence the infimum and supremum [(128), (129)] coincide,

$$
a=b=\left(1-r z_{0}\right) /(1-r)<1
$$

and therefore we have the following exact expression

$$
\lim _{k \rightarrow x} \operatorname{Pr}\left\{X_{k}>n\right\}=\left(1-r z_{0}\right) z_{0}{ }^{-n} /(1-r) .
$$

Furthermore, an upper bound for $p^{(L)}(L)$ is obtained, from (133),

$$
p^{(L)}(L) \leqslant\left(1-r z_{0}\right) z_{0}{ }^{1-L} /(1-r) .
$$

Sometimes the quantity of principal interest may be the expected overflow $Q(L)$ [data units] from the buffer at each time unit, or the average fraction $R(L)$ of the input which is lost due to buffer overflow. These quantities will be given in terms of $p^{(L)}(n)$ as follows:

$$
Q(L)=\sum_{0 \leqslant m<L} p^{(L)}(L-m) \sum_{0 \leqslant k<\infty} k h_{m+k}
$$

and

$$
R(L)=Q(L) / E\left\{\alpha_{k}\right\} .
$$

We can then derive [151] exponentially tight upper and lower bounds of the form (140) for the quantities $Q(L)$ and hence $R(L)$ as well:

$$
Q(L) \sim q(L) z_{0}-L
$$

with

$$
q_{0} \leqslant q(L) \leqslant q_{1}<\infty .
$$

\section{MULTIPLEXING NETWORKS}

We continue with our examination of discrete queueing models, in this section focusing on specific applications. The term multiplexing networks is used in connection with computer communication networks which employ some variant of time-division multiplexing to share communication channels. We have three network structures in mind-the star network (Fig. 5), the loop network (Fig. 6), and the multidrop network (Fig. 7).

By time-division multiplexing we mean that the time axis $0 \leqslant t<\infty$ is divided into contiguous intervals (called slots or frames) $s_{j}=[(j-1) \Delta, j \Delta)$ of some fixed length $\Delta$. The slot length is sufficient to transmit one unit of information (a data unit or packet) which may be a byte, character, or block of characters of fixed length. The size of the slot depends upon the unit of information and the line speed. In each of the three networks data are transmitted to (or from) the master node, the host computer, from (or to) the slave nodes, the terminals. The channels may be either full-or half-duplex. The simplest data network is the star network (of Fig. 5). Each terminal is linked to the host computer by a dedicated channel. It is the usual configuration by which terminals are linked (via modems) to a processor.

For each of the three networks shown in Figs. 5-7 we will assume that the arrival of messages at a terminal is described by a renewal process $\mathrm{A}_{i}=\left\{A_{i, j}: j=1,2, \cdots\right\}$ where $A_{i, j}$ is the number of data units entering the buffer at $i$ th terminal in the interval $(j-1) \Delta \leqslant t<j \Delta$. For notational convenience we will henceforth take units so that $\Delta=1$. We assume that the $n$ arrival processes $\left\{\mathbf{A}_{i}\right\}$ are independent. The state of each of these systems will be described by an $n$-dimensional vectorvalued variable $X_{j}=\left(X_{1, j}, \cdots, X_{n, j}\right)$ where $X_{i, j}$ is the number of data units buffered at the $i$ th terminal at time $j-0$. In a series of papers Konheim and Meister [89]- [92], Konheim [93], [94], and Chu and Konheim [23], various properties of these networks were explored. The analyses have a common theme to which we now turn. To simplify the presentation, we will consider only the case of data flowing from a terminal to the host computer.

We begin with the star network. The evolution of the random process $X$ of the state of the system is given by

$$
X_{i, j}=\left(X_{i, j-1}-1\right)^{+}+A_{i, j}
$$

where $a^{+}=\max (a, 0)$. Note that $(149)$ reflects the fact that a data unit is removed from the buffer at the $i$ th terminal and transmitted to the host computer at time $(j-1)$, provided that the buffer contained a data unit, $\left(X_{i, j-1}>0\right)$ leaving 


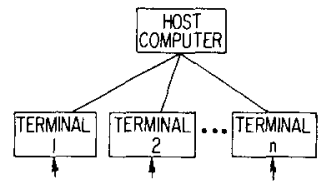

Fig. 5. Star network.

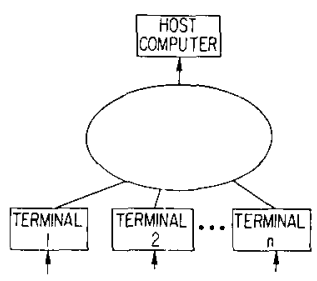

Fig. 6. Loop network.

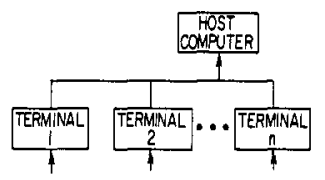

Fig. 7. Multidrop network.

$\left(X_{i, j-1}-1\right)^{+}$data units remaining in the buffer which are joined by $A_{i, j}$ new arrivals during the interval $[(j-1), j)$. The important observation to make from (149) is that the $n$ random processes $X_{i}=\left\{X_{i, j}\right\}(1 \leqslant i \leqslant n)$ are independent. Since each terminal has a dedicated channel to the host computer there is no contention for this resource. We define the pgf of the variable $X_{i, j}$

$$
H_{i, j}(z)=E\left\{z_{i, j}{ }^{X}\right\}=\sum_{0 \leqslant k<\infty} \operatorname{Pr}\left\{X_{i, j}=k\right\} z^{k} .
$$

Then for a given terminal $i$, the generating function of the time sequence $\left\{H_{i, j}(z)\right\}$ is defined by

$$
H_{i}(z, w)=\sum_{0 \leqslant j<\infty} H_{i, j}(z) w^{j}
$$

with $|z|,|w| \leqslant 1$, this condition guaranteeing that the series in (150), (151) converge. The evolution equation (149) translates into the algebraic recursions

$$
\begin{aligned}
H_{i, j}(z)= & H_{i, j-1}(0) P_{i}(z)+\left[H_{i, j-1}(z)\right. \\
& \left.-H_{i, j-1}(0)\right] P_{i}(z) / z, \quad 1 \leqslant j<\infty .
\end{aligned}
$$

From (152) and (151) we then have

$$
H_{i}(z, w)=\left[z H_{i}(z, 0)+P_{i}(z)(z-1) w H_{i}(0, w)\right] /\left(z-w P_{i}(z)\right)
$$

where $P_{i}(z)$ is the pgf of the arrival process $\mathrm{A}_{i}$ at the $i$ th terminal,

$$
P_{i}(z)=E\left\{z_{i, j}^{A}\right\}=\sum_{0 \leqslant k<\infty} \operatorname{Pr}\left\{A_{i, j}=k\right\} z^{k}
$$

Note that $P_{i}(z)$ is independent of the time index $j$ since we have assumed that the arrival process $A_{i}$ is a sequence of iid random variables. Note also that (152) provides a recursive solution; given an "initial" condition $H_{i, 0}(z)$ we may obtain by successive applications of (152) the distribution of the state at succeeding times. The solution given in (153) contains an "undetermined" term $H_{i}(0, w)$. This term is found by making use of the fact that $H_{i}(z, w)$ is analytic in the region $|z|,|w|<$ 1. According to Rouchés theorem [3], the denominator of (153) has a unique zero $\vartheta_{i}(w)$ with $\left|\vartheta_{i}(w)\right|<1$ :

$$
\vartheta_{i}(w)-w P_{i}\left(\vartheta_{i}(w)\right)=0
$$

for each $w,|w|<1$. The function $\vartheta_{i}(w)$ has an important probabilistic interpretation. Assume that the system starts with no customers waiting or in service, $X_{i, 0}=0$ and let $\left\{T_{i, s}: 0 \leqslant s \leqslant \infty\right\}$ denote the times at which $X_{i, j}=0$. Thus $X_{i, T_{s}}=0$ and $X_{i, j} \neq 0$ for $T_{i, s-1}<j<T_{i, s}$. The times $\left\{T_{i, s}\right\}$ are the slot numbers at which the $i$ th terminal is empty and does not require service. The intervals between these epochs $\tau_{i, s}=T_{i, s}-T_{i, s-1}(1 \leqslant s<\infty)$ constitute a renewal process; $\vartheta_{i}(w)$ is the generating function of the probability distribution $\operatorname{Pr}\left\{\tau_{i, s}=k\right\}$

$$
\vartheta_{i}(w)=\sum_{0 \leqslant k<\infty} \operatorname{Pr}\left\{\tau_{i, s}=k\right\} w^{k} .
$$

If $H_{i}(z, w)$ is to be analytic, it follows that the numerator of (153) must vanish when $z \rightarrow \vartheta_{i}(w)$ and we obtain the relation

$$
\begin{aligned}
0 & =\vartheta_{i}(w) H_{i}\left(\vartheta_{i}(w), 0\right)+w P_{i}\left(\vartheta_{i}(w)-1\right) H_{i}(0, w) \\
& =\vartheta_{i}(w)\left[H_{i}\left(\vartheta_{i}(w), 0\right)+\left(\vartheta_{i}(w)-1\right) H_{i}(0, w)\right] .
\end{aligned}
$$

We claim that we must assume $P_{i}(0)=\operatorname{Pr}\left\{A_{i, j}=0\right\}>0$; for otherwise, $A_{i, j} \geqslant 1$ (that is, at least one arrival during each slot interval) and this implies that the queueing process is transient. In physical terms, customers arrive too fast for the service capacity of the system. In mathematical terms, $X_{i, j} \rightarrow$ $\infty$ as $j \rightarrow \infty$ and the process $\mathbf{X}_{i}=\left\{X_{i, j}\right\}$ is transient. If $P_{i}(0) \neq 0$, then $\vartheta_{i}(w) \neq 0$ for $w \neq 0$ and (156) yields

$$
H_{i}(0, w)=H_{i}\left(\vartheta_{i}(w), 0\right) /\left(1-\vartheta_{i}(w)\right) .
$$

By inversion of the generating function [using (153) and (157)] we obtain the behavior (as a function of time) of the fluctuations of the contents of the buffer at the $i$ th terminal. Usually one is interested in more modest information; the stationary (or limiting) behavior.

$$
\lim _{j \rightarrow \infty} H_{i, j}(z)=H_{i}^{*}(z)(\text { say }) .
$$

This limit may be obtained from (153) using a Tauberian argument. One can prove that

$$
\lim _{w \rightarrow 1} H_{i}(z, w)(1-w)=\lim _{k \rightarrow \infty}(1 / k) \sum_{0 \leqslant j<k} H_{i, j}(z)=H_{i}^{*}(z) .
$$

The middle limit in (158) is called the Ceasaro- 1 limit of the sequence $\left\{H_{i, j}(z)\right\}$. It is a time average and the coefficients of the generating function $H_{i}^{*}(z)$

$$
H_{i}^{*}(z)=\sum_{0 \leqslant k<\infty} c_{i, k} z^{k}
$$


may be interpreted as the limiting fraction of time that the state of the system is $k$. Applying the Tauberian theorem we obtain the pgf

$$
H_{i}^{*}(z)=\left(1-\mu_{i}\right)\left[(z-1) P_{i}(z)\right] /\left(z-P_{i}(z)\right) .
$$

As contrasted with a star network, the single channel of the loop network is shared by many users thus offering possible economies of operation. A loop network is an example of a queueing system in which a resource, in this case the channel, is shared by many customers. To specify a loop system we must describe how this sharing is accomplished, in the language of queueing theory, the service or queue discipline. A queue discipline is essentially an allocation of the set of slots. If $\Omega=\{1,2, \cdots\}$ denotes the set of slots, a queue discipline partitions $\Omega$ into $n$ subsets $\Omega=\cup_{1 \leqslant i \leqslant n} \Omega_{i}$. The partitioning may be static-time- or state-independent-or "adaptive" depending upon the state of the system. Here are some examples of possible allocations.

Synchronous Time-Division Multiplexing (STDM): Each user (terminal) is given a fixed (static) assignment of slots. For example,

$$
\Omega_{i}=\{i, i+n, i+2 n, \cdots\} .
$$

Every $n$th slot is assigned to the $i$ th user. More generally, if the users have widely differing rates of sending information to the host computer we might want to give different "proportions" of the total set of slots to different users, in effect, to match the service to the demands of the users.

Asynchronous Time-Division Multiplexing (ATDM): The $n$ users are located on a channel and have a natural priority determined by their position on the loop. Service will be in accordance with this positional priority structure. The "first" terminal (in the sense of position) which requires a slot "grabs it." Hence the channel is multiplexed asynchronously with respect to the users. We may introduce a special priority structure on the loop with ATDM replacing the natural priority determined by position. A slot grabbed by the $i$ th terminal may be retaken by the $j$ th terminal $(j>i)$ if the priority structure so dictates.

Polling: Polling refers to a service discipline which may be applied in the star, loop, and multidrop networks. By a poll of a terminal we mean an inquiry, made to determine if the terminal wishes to transmit data to the host computer. A polling service discipline consists of sequentially polling the terminals. Each terminal is allowed to send the entire contents of its buffer to the host computer. After completion of this operation, control is returned to either the host computer (as in the star) or (as in the loop) to the next terminal. The usual polling cycle is $\{1,2, \cdots, n\}$-the terminals being polled in the order $1,2, \cdots, n-$ and is then repeated. More generally, we may extend the notion of a polling cycle by allowing repeated polls of a terminal in the cycle in order to "match" the service offered to the service required.

Chaining: IBM's Synchronous Data Link Control (SDLC) loop allows for the chaining of messages. The strategy is similar to polling except that we do not allow the terminal to empty its buffer. Terminals are polled sequentially. A cycle is a poll of each of the $N$ terminals. During each cycle a terminal is allowed to send up to a fixed number of data units (bytes, packets, characters). Messages are thus sent as a chain of segments to the host computer.

These service policies and others for the loop network have been reported on in several papers. In Chu and Konheim [23] a survey of various service disciplines is given; an analysis of STDM with equal slot allocations is presented. In an unpub. lished master's thesis at New York University, McKee investigated the case of STDM with variable slot allocation. ATDM was analyzed in Konheim and Meister [89], Hayes and Sherman [57], [58], Avi-Itzhak [5] , and in a survey paper of Konheim [93]. Polling (which in the queueing literature is often referred to as alternating priority queues) has been considered by Eisenberg [35], Hashida [56], [57], Cooper and Murray [27], and Konheim and Meister [92]. Pawlikowski [119], [120] has studied various service policies for the loop network. Wu and Chen [150] and Konheim [94] have given analyses of the chaining discipline.

Space does not permit a complete review of the analysis of each of these queueing disciplines. We will indicate only the central ideas. We begin by showing how the analysis of the star network may be translated to give information about the queueing discipline ATDM. The state of the system and the arrival processes are as defined before. The equations of evolution of the loop system under the discipline ATDM-the analog of (149)-are

$$
\begin{gathered}
X_{i, j}=\left(X_{1, j-1}-1\right)^{+}+A_{1, j}, \\
1 \leqslant j<\infty \\
\left.X_{i, j}=\left(X_{i, j-1}-\chi_{\left(X_{1, j-1}=\cdots\right.}=X_{i-1, j-1}=0\right)\right)^{+}+A_{i, j}, \\
1<i \leqslant n, 1 \leqslant j<\infty .
\end{gathered}
$$

We make the following observations. 1) The equation of evolution for the first terminal on the loop is the same as in the star network. The first terminal does not "feel" the presence of the remaining $n-1$ terminals. 2) the $i$ th terminal can seize a slot only if this slot has not been previously taken by one of the first $i-1$ terminals; that is, the $(j-1)$ st slot is available to the $i$ th terminal if and only if $X_{1, j-1}=X_{2, j-1}=\cdots=X_{i-1, j-1}=$ 0 .

The system of equations (160), (161) can be looked at from at least two points of view. First, set

$$
\begin{aligned}
& Y_{i, j}=X_{1, j}+\cdots+X_{i, j} \\
& B_{i, j}=A_{1, j}+\cdots+A_{i, j}
\end{aligned}
$$

and note that (160), (161) can be rewritten as

$$
Y_{i, j}=\left(Y_{i, j-1}-1\right)^{+}+B_{i, j}, \quad 1 \leqslant i \leqslant n, 1 \leqslant j<\infty
$$

Note that (162) is identical to (149) provided we make the identification of variables

$$
X_{i, j} \leftrightarrow Y_{i, j} A_{i, j} \leftrightarrow B_{i, j} .
$$


Thus the distribution of the total number of data units buffered at the first $i$ terminals can be calculated by, reinterpreting the results for the star network. In particular, by replacing $P_{i}(z)$ and $\mu_{i}$ in $(159)$ by $\Pi_{1 \leqslant k \leqslant i} P_{k}(z)$ and $\Pi_{1 \leqslant k \leqslant i} \mu_{k}$, respectively, we obtain the generating function

$$
\lim _{j \rightarrow \infty} E\left\{z_{i, j} Y\right\}
$$

A second and more revealing method of analysis starts with an examination of the epochs $\left\{T_{1, s}: 0 \leqslant s<\infty\right\}$ at which the first terminal does not require a slot. These are the epochs at which the slot is available for seizure by the second terminal. As we have noted, these interseizure times $\left\{\tau_{1, s}=T_{1, s}\right.$ $\left.T_{1, s-1}\right\}$ form a renewal process and the equation of evolution for the second terminal can be written as

$$
X_{2, T_{s}}=\left(X_{2, T_{s-1}}-1\right)^{+}+C_{2, s}, \quad 1 \leqslant s<\infty
$$

where $C_{2, s}=A_{2, T_{s-1}+1}+\cdots+A_{2, T_{s^{\prime}}} C_{2, s}$ is the total num. ber of data units which enter the buffer at the second terminal in the time interval $\left(T_{s^{-1}}, T_{s}\right]$. The arrival process $\left\{C_{2, s}\right\}$ is a compound process with generating function $\vartheta_{1}\left(P_{2}(x)\right)$. Thus the generating function of the stationary distribution of the variables $\left\{X_{2, T}\right\}$ is obtained from (149) if we replace $P_{i}(z)$ and $\mu_{i}$ by $\vartheta_{1}\left(P_{2}(z)\right)$ and $\mu_{2} /\left(1-\mu_{1}\right)=\left.(d / d z) \vartheta_{1}\left(P_{2}(z)\right)\right|_{z=1}$, respectively. Succeeding terminals can be analyzed in the same manner; for the third terminal we need to know the epochs at which the first two terminals do not require a slot. This requires the determination of a $\vartheta$-function which we obtain by solving equation (162) for $i=2$. Details can be found in [92].

Clearly the ATDM discipline offers better service to terminals at the "start" of the loop. If the service requests (as measured, say, by the data rates $E\left\{A_{i, j}\right\}$ ) are nonincreasing in $i$, then the terminals are "matched" with the service discipline. The first terminal, which sends data at the highest rate, receives preferred service. In [66] a priority discipline was introduced to "correct" a possible mismatching of position and service requirements when these data rates fail to be nonincreasing. This priority discipline for the loop essentially "moves" the position of the terminal and partially mitigates the effect of position on service.

Our discussion has been limited so far to transmission from a terminal to the host computer. It is possible to model twoway traffic. The host computer may be viewed as just another terminal competing with other users of the channel for service. Various models for two-way communication are discussed in [90], [91].

We conclude with a brief discussion of the queueing discipline polling. Polling may be applied to any of the three network structures; the differences are in the way control is passed from terminal to terminal. In the star network, the host computer must poll each of the terminals; in the loop and multidrop network terminals may sense that they are now able to sieze the channel and thus in a sense the control of the server is distributed. We continue with the state description $\mathbf{X}=\left\{X_{j}=\left(X_{1, j}, \cdots, X_{n, j}\right)\right\}$ and introduce the generating func. tion (with $n$ variables)

$$
F_{j}\left(z_{1}, z_{2}, \cdots, z_{n}\right)=E\left\{z_{1}{ }^{X_{1, j} z_{2}}{ }^{X_{2, j} \cdots} z_{n}{ }^{\left.X_{n, j}\right\}}\right. \text {. }
$$

Under polling, each of the terminals is interrogated and allowed to transmit all buffered data units to the host computer, A terminal holds the channel until it empties its buffer. A poll of each of the $n$ terminals is a cycle. After the terminal empties its buffer, it indicates this by transmitting an end of message (EOM) character. Then the channel may be taken by the host computer in order to transmit data to the terminals. There may also be a switching time to transfer control to the next terminal.

The process $\mathbf{X}$ is not Markovian and the state space description must be augmented to make it Markovian. However, there exists a sequence of "times" (slot numbers)

$$
0=\eta_{1,1}<\cdots<\eta_{n, j-1}<\cdots<\eta_{n, j}<\eta_{1, j+1}<\cdots
$$

which are regeneration points of the process $X . \eta_{i, j}$ is the time in the $j$ th cycle at which control is given to the $i$ th terminal. The embedded process obtained by observing $\mathbf{X}$ only at these times is a Markov chain. Let $G_{i, j}\left(z_{1}, \cdots, z_{n}\right)$ be the generating function of the vector variable $X_{\eta_{i, j}}$. The evolution of the system can be described in terms of cyclic shift operators $\left\{\Omega_{i}\right\}$ which change (operate on) the functions $G_{i, j}$

$$
\begin{aligned}
& \Omega_{i}: G_{i, j}\left(z_{1}, \cdots, z_{n}\right) \rightarrow G_{i+1, j}\left(z_{1}, \cdots, z_{n}\right), \quad 1 \leqslant j<n \\
& \Omega_{n}: G_{n, j}\left(z_{1}, \cdots, z_{n}\right) \rightarrow G_{1, j+1}\left(z_{1}, \cdots, z_{n}\right) .
\end{aligned}
$$

The operator $\Omega=\Omega_{n} \cdot \cdots \cdot \Omega_{1}$ corresponds to a single cycle in the sense that

$$
\Omega: G_{1, j}\left(z_{1}, \cdots, z_{n}\right) \rightarrow G_{1, j+1}\left(z_{1}, \cdots, z_{n}\right) .
$$

This functional notation is perhaps less familar than the usual matrix formulation in which we describe the evolution (of the state probabilities) by multiplication by the transition matrix. Equations (166) and (167) show that we are dealing with a nonstationary Markov chain. If we choose to observe the process $X$ only at the start of a cycle then this embedded process is stationary; its transition matrix has been expressed in terms of the operator $\Omega$. A stationary solution is then a function $G^{*}$ $\left(z_{1}, \cdots, z_{n}\right)$ which satisfies

$$
\Omega G^{*}=G^{*} .
$$

(Compare this with the usual condition that the stationary distribution $\pi$ should satisfy the matrix equation $\pi M=\pi$ ). In [91] it is shown how the solution of (168) may be obtained. There is one additional point worth mentioning. We have obtained a solution for the embedded process. How does it relate to the original process? For example, what shall we mean when we ask for the "average number of data units buffered at the first terminal?" From the probability generating function of the stationary distribution $G^{*}$ of the embedded chain we may calculate

$$
\nu_{1}=\left.\left(\partial / \partial z_{1}\right) G^{*}\left(z_{1}, \cdots, z_{n}\right)\right|_{z=1}
$$


the expected number of data units buffered at the first terminal at the start of a cycle. On the other hand, the usual meaning of the average number of data units buffered at the first terminal is the time-average

$$
\nu_{1}=\lim _{n \rightarrow \infty}(1 / n) \sum_{0 \leqslant k<n} X_{1, k}
$$

Whereas $\nu_{1}$ is an average over all time points $v_{1}$ is an average over a subsequence of times

$$
v_{1}=\lim _{n \rightarrow \infty}(1 / n) \sum_{0 \leqslant k<n} X_{1, \eta_{1, k}}
$$

The two time averages (171) and (170) are proportional; the proportionality factor requires the computation of

$$
\left.\left(\partial^{2} / \partial z_{1}^{2}\right) G^{*}\left(z_{1}, \cdots, z_{n}\right)\right|_{z=1} .
$$

The major technical difficulty in obtaining a solution for the polling discipline is related to the calculation of the derivative in (172). Details again are found in [92].

\section{CONCLUDING REMARKS}

The results of Sections III and IV obtained for a class of general Markovian queueing systems are characterized by two major properties: 1) decomposability and 2) robustness. The class of models discussed there is, in fact, the largest set of queueing system for which we know simple closed-form solutions. The "separation of variables" technique is applicable to the system equations defined over multidimensional queueing processes. The solution results are robust in the sense that: 1) they are insensitive to distributional forms of service time when the discipline is PS or LIFO with preemptive resume, or the service station has ample parallel servers, 2) they are also invariant under queue disciplines-any work-conserving discipline-in the case of exponential service distributions, and 3) they do not depend on the detailed behavior of job or message routing.

Despite such generality and simplicity, there are several limitations we must be aware of in attempting to use a queueing network model. They are 1) the message independence assumption; 2) insufficient information about response time; 3) limited results on the finite capacity case; and 4) assumption that the service distribution for FIFO stations is exponential.

The message independence assumption was briefly mentioned at the end of Section III. If IMP's of a computer network are represented as servers in a queueing network mode, this independence assumption amounts to choosing the length of a given message independently at different servers (IMP's) through which the message passes. In reality, however, the message length remains unchanged, since it carries a fixed constant amount of information. Thus a message is transmitted without resampling whereas the model assumes that it is trans- mitted with resampling. Rubin [132], [133] has removed this independence assumption in his model of a packet-switching communications network. The model, however, requires some restrictions on both network topology and message flow conditions.

As for 2) above, analytic solutions for the waiting and response time distributions are still an open problem. Note, however, that the average waiting and response times can be obtained easily from Little's formula $L=\lambda W$. The main difficulty in obtaining the full distribution is due to the fact that the waiting time in successive visits of a customer to the same station are not independent, since the queue-size functions are time-correlated processes. It seems plausible to formulate the problem as a first-passage-time in a Markov system, but to the best of our knowledge it has not been solved.

The situation with a finite waiting room presents problems of various degrees of difficulty. If the total population of the network $\|K\|$ is limited to, say $L$, and the excessive arrivals from the outside are prohibited (or lost), we can model this situation by letting the queue-dependent arrival rate $\lambda(\|K\|)$ vanish for $\|K\|>L$. If, on the other hand, a population constraint is imposed on a subnetwork or on an individual server, an additional arrival may be blocked from entering. Closed. form solutions are difficult to obtain. In [95] a two-stage tandem network with feedback, exponential service, and Pois son arrival was studied. The second stage server has a finite waiting room which, when full, blocks the first server. The stationary state probabilities were obtained from a detailed examination of the two-dimensional generating functions of the process. Both Chang [16] and Neuts [110] have studied the same blocking phenomena in cascaded queues. If one is willing to resort to numerical methods, one can represent the entire system as a multidimensional Markovian system and solve the matrix equation to obtain the stationary solution. See Wallace [144] and references cited in this paper for efficient computational algorithms.

Finally the question raised in 4) can be reduced also to a blocking problem. As stated earlier, a service station with nonexponential service time distribution can be approximated arbitrarily closely in terms of a serial and parallel combination of exponential servers (or alternately, by means of Cox's representation [30] which uses complex probabilities). This fictitious representation of a general server can be viewed as a queueing network; the number of customers in this fictitious queueing network is limited to at most one. This condition is tantamount to blocking the exponential server of the first stage, while a customer is somewhere in this fictitious network.

In order to alleviate the difficulty involved with general service time distributions, an approximate representation of a jump process (like the queue-size process) by a diffusion process -a continuous time (continuous path) Markov processhas recently been investigated. The diffusion approximation for the $G I / G / 1$ system is discussed in the monograph of Newell [113]. Gaver and Shedler [46] applied this technique to the analysis of a multiprogrammed system modeled as a two-stage cyclic queueing network. Kobayashi [82], [83] considered 
the multidimensional diffusion approximation as a technique for treating the general queueing network. An application of the diffusion approximation to the performance analysis of an ALOHA-like system may be found in the paper by Kobayashi, Onozato, and Huynh [87].

The diffusion approximation is often adopted in heavy traffic theory (Kingman [71], Whitt [147]) since the accuracy of this approximation model is generally good under heavy traffic conditions. The main idea behind this technique is as follows; the number $N$ of events (say arrivals) which are observed in a given point process (arrival process) during an interval of length $\Delta$ is well approximated by a Gaussian random variable provided $\Delta$ is sufficiently large. This asymptotic property is in a dual relationship to the central limit theorem which can be stated, in our context, as follows; the random length $\Delta$ of the interval we must wait to observe some fixed number of events $N$ has approximately the Gaussian distribution provided $N$ is sufficiently large. The diffusion approximation can be viewed as a second-order approximation in the sense that the diffusion approximation reflects the trend of the mean (firstorder statistic) and variance (second-order statistic) of the jump process. If we ignore the variance, then we have the socalled fluid approximation that essentially amounts to treating a flow of customers as a flow of fluid. See Newell [113], [114] for a discussion of this subject and related graphical representation. Kleinrock and Lam [81] discuss the stability problem of a slotted ALOHA system based upon the fluid approximation approach. See also a survey article by Kobayashi [86] for a discussion of the stability and control problems for a virtual-storage muitiprogrammed system which is also formulated as a fluid approximation problem.

Chandy, Herzog, and Woo [13], [14] proposed an iterative method for the approximate analysis of a queueing network with general service time distributions. For a general network of queues, they consider a "near-equivalent" network of exponential servers with the same topological structure as the original network. The criteria of the near equivalence are: 1) the sum of the average queue size in the exponential network model is close to the true value in the original system; and 2) the throughput of the individual servers should satisfy the "conservation of flows" rule. The exponential model is allowed to have queue-dependent service rates in meeting these requirements.

One of the remaining tasks in both the iterative approximation technique and the diffusion approximation method is to establish some general formula which allows us to assess the accuracies of these two approximate solutions. For a discussion of the decomposition of the general network of queues, the reader is directed to the paper by Cherry and Disney [17].

In connection with approximate methods, bounds for waiting time distributions or other quantities offer problems for research of both mathematical depth and practical importance. We noted in Section $\mathrm{V}$ that the exponentially tight bounds for the $G I / G / 1$ system were applicable to the study of a broad class of buffer overflow problems. It will also be instructive to point out that the characteristic roots $\left(\vartheta_{0}\right.$ and $z_{0}$ ) which appear in those bounds could be derived from the diffusion approximation solution [71], [84]. The bounds become tighter as the traffic increases.

One important issue, almost exclusively excludẹd from our presentation, is the question of priority and scheduling. Priority queues treated in most books are for the $M / G / 1$ system with multiclass customers, in which the job class is based upon some external priority. That is, the priority level or value of a job is determined externally and before the job enters the system. There are two classes of scheduling rules in priority system; preemptive and nonpreemptive. General solutions have been obtained for the waiting time distributions in the $M / G / 1$ system with multiclass customers. The solutions, however, are expressed as Laplace transforms and it is often tedious to calculate even the second moment of the waiting time. Other types of priority are defined in terms of what we sometimes called the "running time" or "dynamic" priority; a job increments or decrements its priority level as a function of elasped time since entry into the system. The CPU scheduling of a time-shared system (and often in a batch-type multiprogrammed system as well) adopts some kind of running time priority and corresponding scheduling rule; for instance, RR, PS, FB, and variants on these. Kleinrock [78] reviews this subject quite thoroughly and gives many references. See also O'Donovan [116]. Analytic solutions have been obtained for the $M / G / 1$ system in terms of the expected response (or waiting) time conditional on the service time of a given job. We should note that these scheduling disciplines are workconserving, if job-switching overhead is ignored. Hence the Poisson input $\rightarrow$ Poisson output property holds if the service times are homogeneous and exponentially distributed. This property holds even for arbitrary service time distributions in the case of PS scheduling as was noted earlier. The notion of priority PS scheduling was introduced by Kleinrock [76], who derived an expression for the conditional expected response time for the $M / M / 1$ system with multiclass customers. O'Donovan [116] recently showed that Kleinrock's results hold for the $M / G / 1$ system with multiclass customers as well.

Chow [18] studied the $M / G / 1$ system with multiclass customers having a state-dependent arrival process. He introduced a new class of nonpreemptive scheduling rules; the server schedules a class-r job with probability $\delta_{r}(X)$ where $X$ is the state of the system and $\delta_{r}(X)$ is a decision function satisfying

$$
\begin{aligned}
\delta_{r}(\mathbf{X}) & \geqslant 0, \quad(1 \leqslant r \leqslant R) \\
\sum_{\mathbf{1} \leqslant r \leqslant R} \delta_{r}(\mathbf{X}) & =1 .
\end{aligned}
$$

Conventional schemes such as head-of-the-line (HOL) and alternating priority amount to choosing the decision function such that $\delta_{r}(X)=1$ for some $r=r_{0}$ and $\delta_{r}(X)=0$ for $r \neq r_{0}$, where $r_{0}$ is a function of the system state $X$. Chow's solution can be combined with the approximation model of Chandy et al. [13], [14] discussed above, when one service stations in a queueing networks adopts a nonpreemptive schedule rule. 
There are a number of other studies on priority queues related to computer communications; see Herzog [62], Gall and Müller [44], Gopinath and Mitra [50], and Marks [103].

\section{REFERENCES}

[1] N. Abramson, "The ALOHA System," in Computer-Communication Networks, N. Abramson and F. F. Kuo, Eds. Englewood Cliffs, NJ: Prentice-Hall, 1973, pp. 501-517.

[2] N. Abramson and F. F. Kuo, Eds., Computer-Communication Networks. Englewood Cliffs, NJ: Prentice-Hall, 1973.

[3] L. V. Ahlfors, Complex Analysis. New York: McGraw-Hill, 1953.

[4] R. R. Anderson et al," "Simulation performance of ringswitched data network," IEEE Trans. Commun., vol. COM20, pp. 576-591, June 1972.

[5] B. Avi-Itzhak, "Heavy traffic characteristics of a circular data network," Bell Syst. Tech. J., vol. 50, pp. 2521-2549, Oct. 1971.

[6] F. Baskett, K. M. Chandy, R. R. Muntz, and F. G. Palacios. "Open, closed and mixed networks of queues with different classes of customers," J. Ass. Comput. Mach., vol. 22, pp. 248-260, Apr. 1975.

[7] U. N. Bhat, "Sixty years of queueing theory," Management Sci., vol. 15, pp. B280-B294, 1969.

[8] P. J. Burke, "The output of a queueing system," Oper. Res., vol. 4, pp. 699-704, Dec. 1956.

[9] -, "Output processes and tandem queues," in Proc. 22nd Int. Symp. Computer-Communication Networks and Teletraffic, Polytech. Inst. of Brooklyn, Brooklyn, NY, Apr. 1972, pp. 419-428.

[10] J. P. Buzen, "Computational algorithms for closed queueing networks with exponential servers," Commun. Ass. Comput. Mach., vol. 16, pp. 527-531, Sept. 1973.

[11] A. B. Carleial and M. E. Hellman, "Bistable behavior of ALOHA-type systems," IEEE Trans. Commun., vol. COM-23, pp. 401-410, Apr. 1975.

[12] K. M. Chandy, "The analysis and solutions for general queueing networks," in Proc. 6th Annu. Princeton Conf. Inf. Sci. Syst., 1972, pp. 224-228.

[13] K. M. Chandy, U. Herzog, and L. Woo, "Păfametric analysis of queueing networks," IBM J. Res. Develop., vol. 19, pp. 3642, Jan. 1975.

[14] -, "Approximate analysis of general queueing networks," IBM J. Res. Develop., vol. 19, pp. 43-49, Jan. 1975.

[15] J. H. Chang, "Terminal response times in data communication systems," IBM J. Res. Develop., vol. 19, p. 43, Jan. 1975.

[16] W. Chang, "Sequential server queues for computer communication system analysis," IBM J. Res. Develop., vol. 19, pp. 272282, May 1975.

[17] W. P. Cherry and R. L. Disney, "Some topics in queueing network theory," in Mathematical Methods in Queueing Theory, A. B. Clarke, Ed. Berlin, Germany: Springer-Verlag, 1974, pp. 23-44.

[18] W.-M. Chow, "Central server model for multiprogrammed computer system with different classes of jobs," IBM J. Res. Develop. vol. 19, pp. 314-320, May 1975.

[19] W. W. Chu, "Buffer behavior for batch Poisson arrivals and single constant output," IEEE Trans. Commun., vol, COM-18, pp. 613-618, 1970 .

[20] W. W. Chu, Ed., Advances in Computer Communications. Dedham, MA: Artech House, 1974.

[21] W. W. Chu, "Demultiplexing considerations for statistical multiplexors," IEEE Trans. Commun., vol. COM-20, pp. 603609, June 1972.

[22] -, "Asynchronous time-division multiplexing," in Computer. Communication Networks, N. Abramson and F. F. Kuo, Eds. Englewood Cliffs, NJ: Prentice-Hall, 1973, pp. 237268.

[23] W. W. Chu and A. G. Konheim, "On the analysis and modeling of a class of computer communication systems," IEEE Trans. Commun., vol. COM-20, pp. 645-660, June 1972.

[24] W. W. Chu and L. C. Liang, "Buffer behavior for mixed input traffic and single control output rate," IEEE Trans. Commun., vol. COM-20, pp. 230-235, 1972.

[25] F. Closs, "Packet arrival and buffer statistics in a packet switching node," in Proc. 3rd Data Communication Symp., St. Petersburg, FL, Nov. 1973, pp. 12-17.

[26] J. W. Cohen, The Single Server Queue. North-Holland, 1969.

[27] R. B. Cooper and G. Marray, "Queues served in cyclic order," Bell Syst. Tech. J., vol. 48, pp. 375-390, 1969.

[28] R. B. Cooper, Introduction to Queueing Theory. New York: Macmillan, 1972.

[29] D. R. Cox, Renewal Theory (Monographs on Applied Probability and Statistics). London, England: Methuen, 1962.

[30] - "A use of complex probabilities in theory of stochastic processes," Proc. Cambridge Phil. Soc., vol. 51, pp. 313-319, 1955.

[31] D. R. Cox and W. L. Smith, Queues. London, England: Methuen, 1958:

[32] D. J. Daley, "Note on queueing output processes," in Mathematical Methods in Queueing Theory, A. B. Clarke, Ed. Berlin, Germany: Springer-Verlag, 1974, pp. 351-354.

[33] D. W. Davies and D. L. A. Barber, Communication Networks for Computers. New York: Wiley, 1973.

[34] J. L. Doob, Stochastic Processes. New York: Wiley, 1953.

[35] M. Eisenberg, "Queues with periodic service and changeover times," Oper. Res., vol. 2, pp. 440-45i, 1972.

[36] D. J. Farber, "A ring network," Datamation, pp. 44-46, Feb. 1975.

[37] D. J. Farber and K. Larson, "The structure of a distributed computer system-The communication system," in Proc. 22nd Int. Symp. Computer-Communication Networks and Teletraffic, Polytech. Inst. of Brooklyn, Brooklyn, NY, Apr. 1972.

[38] W. D. Farmer and E. E. Newhall, "An experimental distributed switching system to handle burtsy computer traffic," in Proc. ACM Symp. Problems on the Optimization of Data Communication Systems, Pine Mountain, GA, Oct. 1969, pp. 1-33.

[39] W. Feller, An Introduction to Probability Theory and Its Applications, vol. II. New York: Wiley, 1966.

[40] F. G. Foster, "On stochastic matrices associated with certain queueing processes," Ann. Math. Statist., vol. 24, pp. 355$360,1953$.

[41] A. G. Fraser, "Spider-An experimental data communications system," in Proc. IEEE Int. Conf. Commun., Minneapolis, MN, June 1974, pp. 21F-1-21F-10.

[42] - "On the interface between computers and data communications systems," Commun. Ass. Comput. Mach., vol. 15, pp. 566-573, July 1972 .

[43] E. Fuchs and P. E. Jackson, "Estimates of distributions of random variables for certain computer communications traffic models," in Proc. ACM Symp. Problems on the Optimization of Data Communication Systems, Pine Mountain, GA, Oct. 1969 , pp. 202-225.

[44] D. A. Gall and H. R. Müller, "Waiting-time distribution and buffer overflow in priority queues systems," IEEE Trans. Commun., vol. COM-20, pp. 865-877, Oct. 1972.

[45] D. P. Gaver, Jr. and P. A. W. Lewis," "Probability models for buffer storage allocation problems," J. Ass. Comput. Mach., vol. 18, pp, 186-198, Apr. 1971.

[46] D. P. Gaver and G. S. Shedler, "Processor utilization in multiprogramming systems via diffusion approximations," Oper. Res., vol. 21, pp. 569-576, 1973.

[47] B. Gavish and A. G. Konheim, "Computer communication via satellites-A queuing model;"' this issue, pp. 140-147.

[48] B. V. Griedenko and I. N. Kovalenko, Introduction to Queueing Theory. Jerusalem, Israel: Israel Program for Scientific Translations, 1968.

[49] B. Gopinath, D. Mitra, and M. M. Sondhi, "Formulas on queues in burst processes-I," Bell Syst. Tech. J., vol. 52, pp. 9-33, 1973.

[50] B. Gopinath and D. Mitra, "Buffering of data interrupted by a source with priority," in Proc. 4th Asilomar Conf. Circuits and Systems, 1970.

[51] W. J. Gordon and G. F. Newell, "Closed queueing systems with exponential servers," Oper. Res., vol. 15, no. 2, pp. 254-265, 1967. 
[52] P. E. Green, Jr., and R. W. Lucky, Eds., Computer Communica. tions. New York: IEEE Press, 1974.

[53] P. E. Green, Jr., and D. T. Tang, "Some recent developments in teleprocessing system optimization," in Proc. IEEE Intercon., vol. 1,1973 , pp. 1-7.

[54] I. Greenberg, "Distribution-free analysis of $M / G / 1$ and $G / M / 1$ queues," Oper. Res., vol. 21, pp. 629-635, Mar.-Apr. 1973.

[55] R. L. Grimsdale and F. F. Kuo, Ed., Computer Communication Networks (NATO Advanced Study Institute Series). Leyden, The Netherlands: Noordhoff, 1975.

[56] O. Hashida, "Gating multiqueues served in cyclic order," Syst. Com. Contr., vol. 1, pp. 1-8, 1970.

[57] -, "Analysis of multiqueue," Rev. Elec. Commun. Lab. Ja. pan, vol. 20, pp. 189-199, Mar.-Apr. 1972.

[58] J. F. Hayes and D. N. Sherman, "Traffic analysis of a ring switched data transmission system," Bell Syst. Tech. J., vol. 50, pp. 2947-2978, Nov. 1971.

[59] _-, "A study of data multiplexing techniques and delay performance," Bell Syst. Tech. J., vol. 51, pp. 1983-2011, Nov. 1971.

[60] J. F. Hayes, "Performance models of an experimental computer communication system," Bell Syst. Tech. J., vol. 53, pp. 225-259, Feb. 1974.

[61] D. Heiman and M. F. Neuts, "The single server queue in discrete numerical analysis IV," Nav. Res. Log. Quart., vol. 20, pp. 753-766, 1973.

[62] V. Herzog, "Optimal scheduling strategies for real-time computers," IBM J. Res. Develop., vol. 19, pp. 494-504, Sept. 1975 .

[63] J. Hsu, "A general queueing model for buffer storage problems," IEEE Trans. Commun., vol. COM-20, pp. 744-747, June 1973.

[64] —, "Buffer behavior with Poisson arrivals and geometric output process," IEEE Trans. Commun., vol. COM-22, pp. 19401941 , Dec. 1974.

[65] J. R. Jackson, "Two shop-like queueing systems," Management Sci., vol. 10, pp. 131-142, 1963 .

[66] S. Katz and A. G. Konheim, "Priority disciplines in a loop system," J. Ass. Comput. Mach., vol. 21, pp. 340-349, Apr. 1974.

[67] A. R. Kaye, "Analysis of a distributed central loop for data transmission," in Proc. 22nd Int. Symp. Computer-Communication Networks and Teletraffic, Polytech. Inst. of Brooklyn, Brooklyn, NY, Apr. 1972, pp. 47-58.

[68] J. Keilson, Green's Function Methods in Probability Theory. New York: Hafner, 1965, pp. 147-172, ch. 6.

[69] D. G. Kendall, "Stochastic processes occurring in the theory of queues and their analysis by the method of imbedded Markov chain," Ann. Math. Statist., vol. 24, pp. 338-354, 1953.

[70] A. Y. Khintchine, Mathematical Methods in Queueing Theory. London, England: Griffen, 1960.

[71] J. F. C. Kingman, "The heavy traffic approximation in the theory of queues," in Proc. Symp. Congestion Theory, W. L. Smith and W. E. Wilkinson, Eds. Chapel Hill, NC: Univ. of North Carolina Press, 1965, pp. 137-159.

[72] - "A martingale inequality in the theory of queues," Proc. Cambridge Phil. Soc., vol. 60, pp. 359-361, 1964.

[73] -, "Inequalities in the theory of queues," Royal Statist. Soc. J., vol. 32, pp. 102-110, 1970.

[74] L. Kleinrock, Communication Nets. New York: McGrawHill, 1964.

[75] -, "A conservation law for a wide class of queueing disciplines," Naval Res. Log. Quart., vol. 12, pp. 181-192, 1965.

[76] --, "Time-shared systems: A theoretical treatment," J. Ass. Comput. Mach., vol. 242-261, Apr. 1967.

[77] -, "Analytic and simulation methods in computer network design," in Proc. 1970 Spring Joint Comput. Conf., AFIPS Conf. Proc., vol. 36. Montvale, NJ: AFIPS Press, 1970, pp. 569-579.

[78] _-., "Scheduling, queueing and delays in time-shared systems and computer networks," in Computer-Communication Networks, N. Abramson and F. F. Kuo, Eds. Englewood Cliffs, NJ: Prentice-Hall, 1973, pp. 95-141.
[79] -, Queueing Systems, Volume 1: Theory. New York: Wiley, 1975.

[80] -, "Performance models and measurements of the ARPA computer network," in Computer Communication Networks (NATO Advanced Study Institute Series), R. L. Grimsdale and F. F. Kuo, Eds. Leyden, The Netherlands: Noordhoff, 1975, pp. $63-88$.

[81] L. Kleinrock and S. S. Lam, "Packet switching in a multiaccess broadcast channel: Performance evaluation," IEEE Trans. Commun., vol. COM-23, pp. 410-423, Apr. 1975.

[82] H. Kobayashi, "Application of diffusion approximation to queueing networks, Part I," J. Ass. Comput. Mach., vol. 21, pp. 316-328, Apr. 1974

[83] --, "Application of diffusion approximation to queueing networks, Part II," J. Ass. Comput. Mach., vol. 21, pp. 459469, July 1974.

[84] -, "Bounds for the waiting time in queueing systems," in Computer Architecture and Networks, E. Gelenbe and R. Mahl, Eds. New York: North-Holland/American Elsevier, 1974.

[85] -, "On discrete-time processes in a packetized communication system," Univ. of Hawaii, Honolulu, Aloha Syst. Tech. Rep. B75-28, Feb. 1975.

[86] _-, "System design and performance analysis using analytic models," in Software Modelling and Its Impact on Performance, K. M. Chandy and R. T. Yeh, Eds. Englewood Cliff, NJ: Prentice-Hall, 1977 (also IBM Res. isep. RA75).

[87] H. Kobayashi, Y. Onozato, and D. Huynh, "An approximate method for design and analysis of an ALOHA system," Univ. of Hawaii, Honolulu, Aloha Syst. Tech. Rep., Mar. 1976; also in this issue, pp. 158-169.

[88] H. Kobayashi and M. Reiser, "On generalization of job routing behavior in a queueing network model," IBM Res. Rep. RC5252, Feb. 1975.

[89] A. G. Konheim and B. Meister, "Service in a loop system," J. Ass. Comput. Mach., vol. 19, pp. 92-108, Jan. 1972.

[90] - "Two-way traffic in loop service systems," Networks, vol. 1, pp. 291-301, 1972.

[91] _-, "Distributions of queue lengths and waiting times in a loop with two-way traffic," J. Comput. Syst. Sci., vol. 7, pp. 506-521, Oct. 1973.

[92] --, "Waiting lines and times in a system with polling," J. Ass. Comput. Mach., vol. 21, pp. 470-490, July 1974.

[93] A. G. Konheim, "Service epochs in a loop system," in Proc. 22nd Int. Symp. Computer-Communication Networks and Teletraffic, Polytech. Inst. of Brooklyn, Brooklyn, NY, Apr. 1972, pp. 125-143.

[94] - "Chaining in a loop system," IEEE Trans. Commun., vol. COM-24, pp. 203-209, Feb. 1976.

[95] _- "An elementary solution of the queueing system G/G/1," SIAM J. Comput., vol. 4, pp. 540-545, Dec. 1975.

[96] A. G. Konheim and M. Reiser, "A queueing model with finite waiting room and blocking," J. Ass. Comput. Mach., to be published.

[97] W. J. Kropfl, "An experimental data block switching system," Bell Syst. Tech. J., vol. 51, pp. 1147-1165, July-Aug. 1972.

[98] A. Kuczma, "The interrupted Poisson process as an overflow process," Bell Syst. Tech. J., vol. 52, 437-447, Mar. 1973.

[99] F. F. Kuo and R. D. Binder, "Computer-communications by radio and satellite: The ALOHA system," in Computer Com. munication Networks (NATO Advanced Study Institute Series), R. L. Grimsdale and F. F. Kuo, Eds. Leyden, The Netherlands: Noordhoff, 1975, pp. 397-408.

[100] P. A. W. Lewis and P. C. Yue, "Statistical analysis of series of events in computer systems," in Statistical Computer Performance Evaluation, W. Freiberger, Ed. New York: Academic, 1972, pp. 265-280.

[101] D. V. Lindley, "The theory of queues with a single server," Proc. Cambridge Phil. Soc., vol. 48, pp. 277-289, 1952.

[102] M. Loéve, Probability Theory. New York: Van Nostrand Reinhold, 1960.

[103] B. I. Marks, "State probabilities of $M / M / 1$ priority queues," Oper. Res., vol. 21, pp. 974-987, July-Aug. 1974.

[104] K. T. Marshall, "Some inequalities in queueing," Oper. Res. vol. 16 , pp. $651-665,1968$. 
[105] J. Martin, System Analysis for Data Transmission. Englewood Cliffs, NJ: Prentice-Hall, 1967.

[106] M. McDonald and H. Rudin, Jr., "Note on inherent and imposed priorities in packet switching," IEEE Trans. Commun., vol. COM-22, pp. 1678-1681, Oct. 1974.

[107] T. Meisling, "Discrete-time queueing theory," J. Oper. Res., vol. 6, pp. 96-105, 1958.

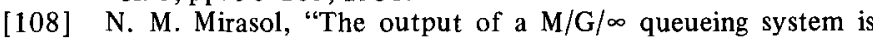
Poisson," Oper. Res., vol. 11, pp. 282-284, 1963.

[109] R. R. Muntz, "Poisson departure processes and queueing networks," in Proc. 7th Annu. Princeton Conf. Info. Sci. Syst., Mar. 1973,pp. 435-440.

[110] M. F. Neuts, "Two queues in series with a finite intermediate waiting room," J. Appl. Prob., vol. 5, pp. 123-142, 1968.

[111] _-, "The single server queue in discrete numerical analysis I," Nav. Res. Log. Quart., vol. 20, pp. 297-304, 1973.

[112] M. F. Neuts and E. Klimko, "The single server queue in discrete numerical analysis III," Nav. Res. Log. Quart., vol. 20, pp. 557-567, 1973.

[113] G. F. Newell, Applications of Queueing Theory. Chapman and Hall, 1971.

[114] _-, "Graphical representation of queues evolution for multipleserver systems," in Mathematical Methods in Queueing Theory, A. B. Clarke, Ed. Berlin, Germany: Springer-Verlag, 1974, pp. $63-80$.

[115] E. E. Newhall and A. N. Venetsanopoulos, "Computer-communications-Representative systems," in Proc. of IFIP 1971. North-Holland, 1971, pp. 545-552.

[116] T. M. O'Donovan, "Direct solution of M/G/1 processor-sharing models," Oper. Res., vol. 22, no. 6, pp. 1232-1235, 1974.

[117] C. D. Pack, "The effects of multiplexing on a computer-communication system," Commun. Ass. Comput. Mach., vol. 16, pp. 161-168, 1973.

[118] - "The optimum design of a random computer buffer in a remote data collection," IEEE Trans. Commun., vol. COM22, pp. 1501-1504, 1974.

[119] K. Pawlikowski, "Queue length distribution in some loop computer network," Bull. Acad. Pol. Sci. Ser. Sci. Tech., vol. XXII, no. 6, pp. $55-62,1974$

[120] —, "Virtual delay distributions in some loop computer networks," Bull. Acad. Pol. Sci. Ser. Sci. Tech., vol. XXII, no. 7, pp. 63-69, 1974.

[121] R. D. Pedersen and J. C. Shah, "Multiserver queue storage requirements with unpacked messages," IEEE Trans. Commun., vol. COM-20, pp. 462-465, June 1972.

[122] J. R. Pierce, "Network for block switching of data," Bell Syst. Tech. J., vol. 51, pp. 1133-1145, July-Aug. 1972.

[123] —, "How far can data loops go?" IEEE Trans. Commun., vol. COM-20, pp. 527-530, June 1972.

[124] F. Pollaczek, "Fonctions caractéristiques de certain es répartitions définies au moyen de la notion d'orde. Application à la théorie des attentes," C. R. Acad. Sci. Paris, vol. 234, pp. 2234-2336, 1952.

[125] E. Reich, "Waiting times when queues are in tandem," Ann. Math. Statist., vol. 28, pp. 768-773, 1957.

[126] _-, "Departure processes," in Proc. Symp. Congestion Theory, W. L. Smith and W. E. Wilkinson, Eds. Chapel Hill, NC: Univ. of North Carolina Press, 1965, pp. 439-457.

[127] M. Reiser and H. Kobayashi, "Queueing networks with multiple closed chains: Theory and computational algorithms," IBM J. Res. Develop., vol. 19, pp. 282-294, May 1975.

[128] - "Horner's rule for the evaluation of general closed queueing networks," Commun. Ass. Comput. Mach., vol. 18, pp. 592-593, Oct. 1975.

[129] J. Riordan, Stochastic Service Systems. New York: Wiley, 1962.

[130] P. N. Robilland, "An analysis of a loop switching system with multirank buffers based on the Markov process," IEEE Trans. Commun., vol. COM-22, pp. 1772-1778, Nov. 1974.

[131] S. M. Ross, "Bounds on the delay distribution in $G I / G / 1$ queues," Dep. Industrial Eng. Oper. Res., Univ. of California, Berekely, rep. ORC 73-1, Jan. 1973.

[132] I. Rubin, "Communication networks: Message path delays," IEEE Trans. Inform. Theory, vol. IT-20, pp. 738-745, Nov. 1974.
[133] —, "Message path delays in packet-switching communication networks," IEEE Trans. Commun., vol. COM-23, pp. 186192, Feb. 1975

[134] H. Rudin, Jr., "Performance of simple multiplexor-concentrators for data communication," IEEE Trans. Commun., vol. COM-19, pp. 178-187, Apr. 1971.

[135] M. S. Schmookler, "Limited capacity discrete time queues with single or bulk arrival," IBM Poughkeepsie Lab., IBM Tech. Rep., TR 00.2048, 1970.

[136] G. D. Schultz, "A stochastic model for message-assembly buffering with a comparison of block assignment strategies," $J$. Ass. Comput. Mach., 1972.

[137] M. Schwartz, R. R. Boorstyn, and R. L. Pickholtz, "Terminaloriented computer-communication networks," Proc. IEEE (Special Issue on Computer Communications), vol. 60, pp. 1408-1422, Nov. 1972.

[138] W. L. Smith, "Renewal theory and its ramifications," J. Roy. Statist. Soc., vol. 20, pp. 243-302, 1958.

[139] J. D. Spragins, "Loop transmission systems-Mean value analysis," IEEE Trans. Commun., vol. COM-20, pp. 592-602, June 1972.

[140] - " "Loops used for data collection," presented at the 22nd Int. Symp. Computer-Communication Networks and Teletraffic, Polytech. Inst. of Brooklyn, Brooklyn, NY, Apr. 1972.

[141] F. Spitzer, "The Wiener-Hopf equation whose kernel is a probability density," Duke Math. J., vol. 24, pp. 327-343, 1957.

[142] E. H. Steward, "A loop transmission system," in Proc. IEEE Int. Conf. Commun., vol. 2, San Francisco, CA, June 1970, pp. 36.1-36.9.

[143] R. Syski, Introduction to Congestion Theory in Telephone Systems. Oliver and Boyd, 1960.

[144] V. L. Wallace, "Algebraic techniques for the numerical solution of queueing networks," in Mathematical Methods in Queueing Theory, A. B. Clarke, Ed. Berlin, Germany: Springer-Verlag, 1974, pp. 295-305.

[145] E. Welman, "A fixed optimum cell-size for records of various lengths," J. Ass. Comput. Mach., vol. 12, pp. 53-70, Jan. 196.

[146] B. A. Whitaker, "Analysis and optimal design of a multiserver multiqueue system with finite waiting space in each queue," Bell Syst. Tech. J., vol. 54, pp. 595-623, Mar. 1975.

[147] W. Whitt, "Heavy traffic limit theorems for queues: A survey," in Mathematical Methods in Queueing Theory, A. B. Clarke, Ed. Berlin, Germany: Springer-Verlag, 1974.

[148] R. W. Wolff, "Work-conserving priorities," J. Appl. Prob., vol. 7, pp. 327-337, 1970.

[149] E. M. Wright, "Solution of the equation $\mathrm{ze}^{\mathrm{z}}=\mathrm{a}$," Bull. Amer. Math. Soc., vol. 65, pp. 89-93, 1959.

[150] R. M. Wu and Y-B. Chen, "Analysis of a loop transmission system with round-robin scheduling of services," IBM Syst. Develop. Div., Internal Rep. TR 21.560, Aug. 1974.

[151] A. D. Wyner, "On the probability of buffer overflow under an arbitrary bounded input-output distribution," SIAM J. Appl. Math., vol. 27, pp. 544-570, Dec. 1974.

[152] M. L. T. Yuen and E. E. Black, "Traffic flow in a distributed loop switching system," in Proc. 22nd Int. Symp. ComputerCommunication Networks and Teletraffic, Polytech. Inst. of Brooklyn, Brooklyn, NY, Apr. 1972, pp. 29-46.

[153] P. Zafiropulo, "Reliability optimization in multiloop communication networks," IEEE Trans. Commun., vol. COM-21, pp. 898-907, Aug. 1973.

Hisashi Kobayashi (S'66-M'68-SM'76) received the B.S. and M.S. degrees in electrical engineering from the University of Tokyo, Tokyo, Japan, in 1961 and 1963, respectively, and the M.A. and Ph.D. degrees in electrical engineering from Princeton University, Princeton, NJ, in 1967.

From 1963 to 1965 he worked for the Toshiba Electric Company, Japan, as a Radar System Analyst. In 1967 he joined the IBM Thomas J. Watson Research Center, Yorktown Heights, NY, where he has been involved in seismic signal processing, data transmission, magnetic re- 


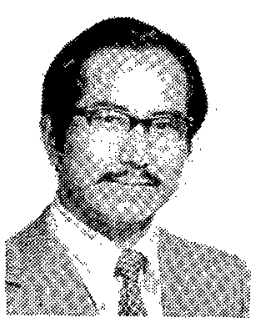

cording image data compression, and modeling and performance analysis of computer systems/ computer communications. He has been manager of the System Measurement and Modeling Group, Computer Science Department, since 1971. During 1969-1970 he was a Visiting Assistant Professor of Systems Science at the University of California, Los Angeles. From July to December 1975 he was a Consultant to the ALOHA Systems Project of the University of Hawaii, Honolulu, and from January to June 1976 he was a Consulting Professor of Stanford University, Stanford, CA, where he taught graduate courses in discrete mathematics and computer performance evaluation.

Dr. Kobayashi is a member of Eta Kappa Nu, ACM, Institute of Electronics and Electrical Communication Engineers of Japan, and Information Processing Society of Japan.

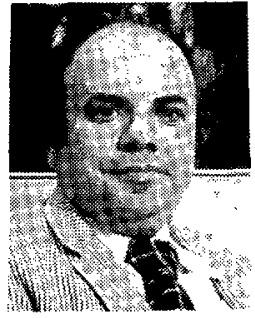

Alan G. Konheim was born in Brooklyn, NY, on October 17, 1934. He received the B.E.E. and M.S. (mathematics) degrees from the Polytechnic Institute of Brooklyn, Brooklyn, NY, and the Ph.D. degree in mathematics from Cornell University, Ithaca, NY, in 1955, 1957, and 1960 , respectively.

Since June 1960 he has been a Research Staff Member of the Mathematical Sciences Department, IBM Thomas J. Watson Research

Center, Yorktown Heights, NY. His principal research interests are in the field of probability theory. He was a Fulbright Scholar at the University of Heidelberg, Heidelberg, Germany, from 1966 to 1967.

Dr. Konheim is a member of the American Mathematical Society, the Mathematical Association of America, and the Society for Industrial and Applied Mathematics.

\title{
Large-Scale Network Topological Optimization
}

\author{
ROBERT R. BOORSTYN, MEMBER, IEEE, AND HOWARD FRANK, MEMBER, IEEE
}

Invited Paper

\begin{abstract}
A cost-effective structure for a large network is a multilevel hierarchy consisting of a backbone network and a family of local access networks. The backbone network is generally a distributed network, while the local access networks are typically centralized systems. In special cases, the network may consist primarily of either centralized or distributed portions. This paper discusses topological design problems for such systems, including the concentrator location problem, the terminal assignment problem, the terminal layout problem (the constrained minimum spanning tree problem), the distributed network topological layout problem, and the backbone node location problem. Recent algorithm research, including exact and heuristic problem solutions, are described and computational experience is given. Finally, open problems in large-scale topological design are reported.
\end{abstract}

\section{INTRODUCTION}

A cost-effective structure for a large network is a multilevel hierarchical structure consisting of a backbone network (high level) and local access networks (low level). The backbone network is characterized by distributed traffic requirements and is generally implemented using packet switching techniques. The backbone network itself may be multilevel, incorporating, for example, terrestrial and satellite channels. Local access networks, on the other hand, have, in general, centralized traffic patterns (most traffic is to and from the

Manuscript received May 13, 1976; revised June 21, 1976. This work was supported in part by the National Science Foundation under Grant GK4347X and in part by the Advanced Research Projects Agency of the Department of Defense under Contract DAHC-15-73C-0135. This paper was presented at the First Joint IEEE-USSR Workshop on Information Theory, Moscow, USSR, December 1975.

R. R. Boorstyn is with the Department of Electrical Engineering, Polytechnic Institute of New York, Brooklyn, NY 11201.

H. Frank is with the Network Analysis Corporation, Glen Cove, NY 11542. gateway backbone node) and are, therefore, implemented with centralized techniques such as multiplexing, concentrating, and polling. The low level local networks may also be hierarchically structured. In special cases, the network may consist primarily of either centralized or distributed portions exclusively.

The topological design problem for a large hierarchical network can be formulated as follows. Given

1) terminal and Host locations,

2) matrix of traffic requirements (terminal-to-Host and Host-to-Host),

3) delay requirements (possibly different requirements for different subsystems)

4) reliability requirements (possibly different requirements for different subsystems),

5) candidate sites for backbone nodes, and

6) cost elements (line tariff structures, nodal processor costs, hardware costs, etc.).

Minimize

Total communications cost $D$ where

$$
\begin{aligned}
D= & (\text { backbone line costs })+\text { (backbone node costs) } \\
& + \text { (local access line costs }) \\
& + \text { (local access hardware costs })
\end{aligned}
$$

such that traffic, delay, and reliability requirements are met.

The global design problem consists of two subproblems: the design of the backbone and the design of the local distribution networks. 